\title{
The Taxonomy and Distribution of the Spider Genus Phryganoporus Simon (Araneae: Amaurobioidea: Desidae)
}

\author{
MiCHAEL R. GRAY \\ Australian Museum, 6 College Street, Sydney NSW 2010, Australia \\ mikeg@austmus.gov.au
}

\begin{abstract}
The widely distributed Australian desid spider genus, Phryganoporus Simon, is taxonomically reviewed and validated. Phryganoporus nigrinus is removed from synonymy with $P$. candidus, while Amaurobius gausapatus, $P$. g. occidentalis and $P$. tubicola are synonyms of $P$. candidus. The species recognised here belong to two clades, [P. candidus (L. Koch), Phryganoporus vandiemeni (Gray) n.comb.] and $[P$. nigrinus Simon, P. davidleei n.sp., P. melanopygus $\mathrm{n} . \mathrm{sp}$.]. These clades are distinguished by the possession of a divided or entire cribellum and genitalic characters. Distribution maps and notes on relationships are given.
\end{abstract}

GraY, Michael R., 2002. The taxonomy and distribution of the spider genus Phryganoporus Simon (Araneae: Amaurobioidea: Desidae). Records of the Australian Museum 54(3): 275-292.

Spiders of the genus Phryganoporus are cribellate web builders that are distributed throughout Australia. They build webs on low vegetation and their abundant white hair cover probably helps reduce body heating, particularly for spiders occupying exposed webs in semi-arid to arid regions.

While the species described here are typically solitary, one species, $P$. candidus, has a life cycle that is associated with both communal and solitary webs. Phryganoporus candidus has long been known for its communal "nest" building behaviour (Main, 1971). In past years the webbing of these spiders upon fruit tree foliage in the Riverina area of New South Wales was a cause of severe foliage matting, leaf fall and withering of limbs. Downes (1993 [Badumna candida] and 1994a,b [Phryganoporus candidus]) has made a detailed study of the biology of $P$. candidus in southern Queensland. He classifies the species as a periodic-social group in which spiders disperse from the parental nest as subadult females and adult males. Most webs were founded in late summer by solitary subadult females. By OctoberNovember, at the peak of colony growth, each nest had about 100 spiders, along with an associated arthropod fauna of opportunist scavengers and predators. Most non-territorial, communal interaction (collective nest construction and cleaning, prey capture and feeding) took place between individuals below the subadult stage. Such communal interaction is probably a consequence of pheromone mediated sibling tolerance and is not regarded as true cooperative behaviour. Subadults males and females are less tolerant of each other. The faster maturing females dispersed over summer, most as subadults. Adult males did not appear in the nest until most of the females had dispersed. By March, the old nests were largely unoccupied.

Simon (1908) erected the genus Phryganoporus and included the following species from southern and western Australia: Amaurobius gausapatus Simon, 1906, P. gausapatus occidentalis Simon, 1908, P. nigrinus Simon, 1908 (all for female specimens) and P. tubicola Simon, 1908 (a male specimen). Roewer (1954) placed Amaurobius candidus L. Koch, 1872 (from Queensland) into Ixeuticus Dalmas and Hickman (1967) followed this transfer. Lehtinen (1967) synonymised Ixeuticus with Badumna Thorell but maintained Phryganoporus into which he placed 
A. candidus (with A. gausapatus and $P$. g. occidentalis as synonyms) and $P$. tubicola. Main (1971) attempted to resolve the apparent taxonomic confusion by synonymising Phryganoporus with Ixeuticus, but recognised only one widely distributed species, Ixeuticus candidus (L. Koch). Gray (1983) synonymised Phryganoporus with Badumna as the B. candida species group made up of three species-B. candida (with $P$. gausapata occidentalis, $P$. tubicola and $P$. nigrinus as synonyms), B. gausapata and B. vandiemeni Gray.

The results of electrophoretic studies by Colgan \& Gray (1992) and more recent morphological examination of new material has prompted a reassessment of the status of Phryganoporus and its associated species.

\section{Materials and methods}

Specimen examinations, measurements (in millimetres) and drawings were made using a Wild M5 microscope with graticule and drawing attachment. Epigynal preparations were cleared in $8 \%$ potassium hydroxide before mounting in glycerol for microscopic examination and drawing of the dorsal view of the internal genitalia. Specimen preparations for scanning electron microscopy were air dried from $100 \%$ acetone.

\section{Abbreviations}

Morphology BL, body length; CL carapace length; CW carapace width; LL, labium length; LW, labium width; SL, sternum length; SW, sternum width; ALS, anterior lateral spinnerets; PMS, posterior median spinnerets; PLS, posterior lateral spinnerets; RTA, retrolateral tibial apophysis.

Repository institutions AMS, Australian Museum, Sydney (specimens listed under accession numbers prefixed KS are deposited in the AMS); ANIC, Australian National Insect Collection, Canberra; MNHN, Museum National d'Histoire Naturelle, Paris; NMV, Museum of Victoria, Melbourne; QM, Queensland Museum, Brisbane; SAM, South Australian Museum, Adelaide; TM, Tasmanian Museum and Art Gallery, Hobart; WAM, Western Australian Museum, Perth; ZMH, Zoologisches Museum, Hamburg.

Collectors M.R. Gray, MRG; B. Guerin, BG; V.V. Hickman, VVH; D. Hirst, DH; C. Horseman, CH; A.E. de Jong, AEJ; D.C. Lee, DCL; G.A. Milledge, GAM; H.M. Smith, HMS; J. Thompson, JT.

Unsexed juvenile specimens are abbreviated $j$.

\section{Taxonomy}

\section{Family Desidae}

\section{Genus Phryganoporus Simon}

Phryganoporus Simon, 1908: 367 (type species Amaurobius gausapatus Simon, 1906). Roewer, 1954: 1370. Lehtinen, 1967: $259,324$.

Ixeuticus (part).-Main, 1971: 119. Brignoli, 1983: 495.

Badumna (part).-Gray, 1983: 248.

Diagnosis. Cribellate spiders. Body silvery grey to brown with light and dark brown patterning on abdomen. In solitary or communal webs. Separated from Badumna by the strong presence of white setae on the body; the possession on the male palp of a large, prolaterally directed, basal tegular bulge; extension of the conductor fold across to the retrolateral side of the bulb; and a basal RTA. From Forsterina and Reinga by the prolateral origin of the embolus and the presence of a transverse epigynal ridge. From Namandia by the sinuous course of the embolus across the ventral bulb and the presence of fused paracribellar spigots on the PMS; and from Paramatachia by these characters plus the presence of a median apophysis.

Description. Medium-sized cribellate spiders (CL 1.9-4.8) that live in solitary and communal sheet webs and run on top of the sheet. Overall colouration varies from silvery grey to brown, depending on the density of white hairs present (juveniles are often lighter in colour than adults). Body setae plumose, comprising shorter, broader, more recumbent hairs, and longer bristle-like hairs (Fig. 1f). Legs with several "plumose" setal types (Fig. 1a-c,i,j); long, vertical, plumose hairs sparsely scattered on lateral and dorsal surfaces. Feathery hairs absent. Carapace cuticle dark brown with a cover of white plumose hairs, giving a silvery grey to brown appearance (older specimens can appear browner through loss of hairs, cf. Fig. 3d). Chelicerae dark brown. Labium and maxillae dark brown with pale apices. Sternum silvery grey to brown, white hairs numerous to few. Abdomen light silvery grey to brown, anterior dorsum usually with a short, dark brown, mid-dorsal stripe, often delimited by a border of white hairs. Posterior dorsum with variable patterns of light and dark brown chevrons that may be more or less fused posteriorly, often with lateral tufts of white hairs. Lateral abdomen with brown flecks, often coalescing into a pair of irregular, longitudinal, brown stripes bordering the venter. Ventral abdomen with a light to dark grey-brown mid-ventral stripe (often enclosing paler patches) flanked by lateral white-pigmented stripes. Spinnerets brown. Legs light brown with darker brown pigment rings often giving a banded appearance. Caput prominent, gently arched; carapace highest near mid-caput. Eyes in two rows, anterior eye row slightly recurved, posterior eye row straight to moderately procurved; AME or ALE largest, lateral eyes slightly protuberant. Tapeta of indirect eyes canoe-shaped. Clypeus low, less than twice as wide as AME. Chilum divided. Chelicerae robust, with boss, fang with serrate retroventral margin; teeth 2-4 retromarginal, 3-5 promarginal; 4-5 moderately long modified setae on retromargin near fang base, about 15 along promargin, longest apically. Maxillae broad, lateral margins more or less convex. Labium short and wide, bluntly coniform, with short basal notches. Sternum longer than wide with a short extension between coxae IV. Legs 1243 or 1423. 3 tarsal claws, superior claws with 7-13 curved teeth, inferior claws with 2-3 teeth. Claw tufts and scopulae absent. Trochanters shallowly notched. Trichobothria in single row on tarsi and metatarsi, rather short but lengthening distally, 5-6 on tarsus I, 4-5 on metatarsus I, 3-4 in short, subdistal row on tarsus and metatarsus IV; trichobothria present on tibiae and on palpal tarsus and cymbium; bothria collariform, proximal plates with fine, longitudinal ridges. Tarsal organ placed subdistally, distal to trichobothria; capsulate with small pyriform pore (Fig. 1a-e). Male palp. Tibia short and wide, with two retrolateral apophyses: an apical ventrad RTA, ventrally protuberant with a narrow, semi-translucent distal margin; and a basal dorsad RTA, robust and spine-like. Inner surface of basodorsal apophysis with 1-2 small trichobothria. Patella 

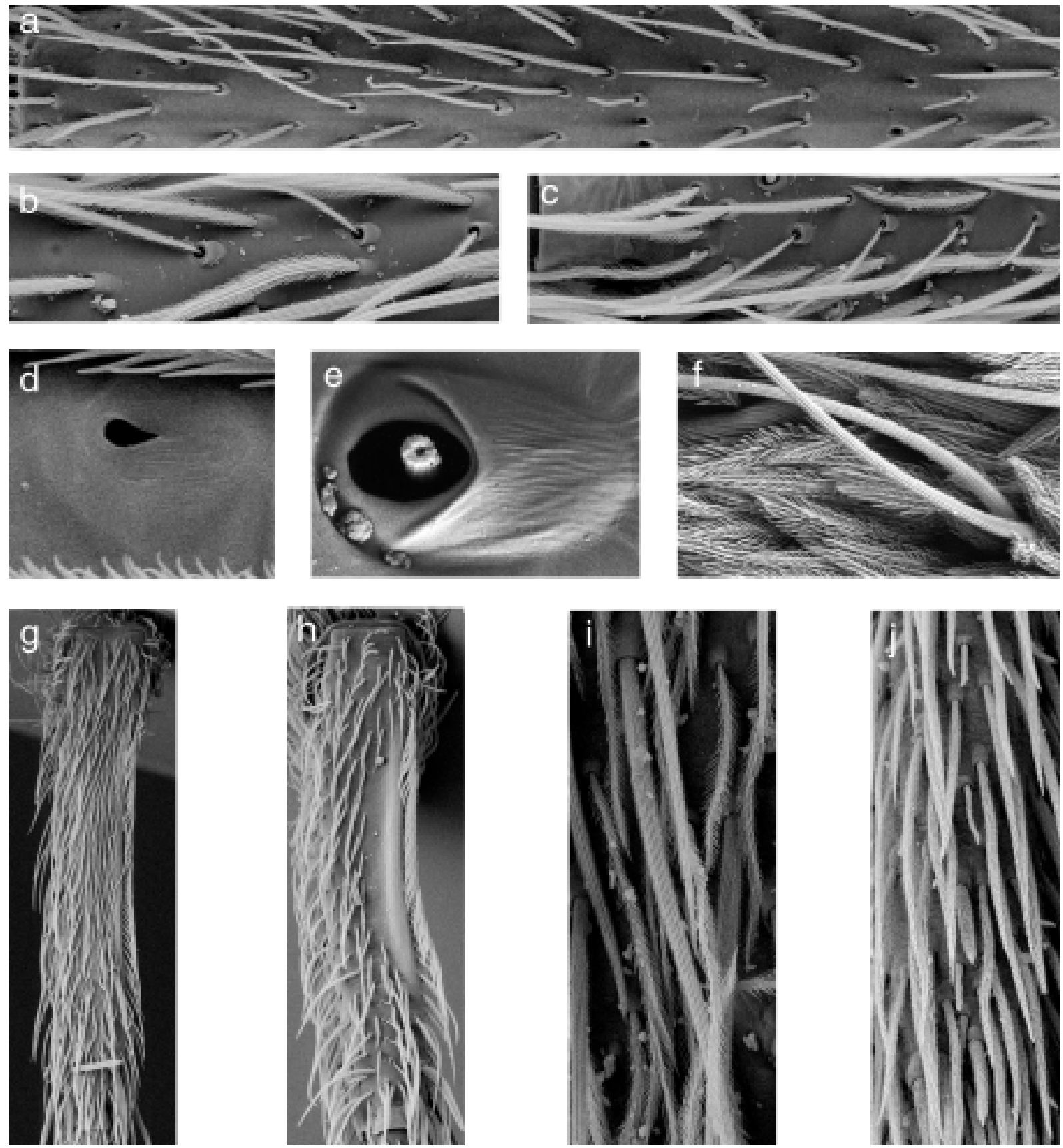

Figure 1. a,g, Phryganoporus candidus. b-f, h-j, Phryganoporus nigrinus. a,b, dorsal tarsi-tarsal organ and trichobothria: a, tarsus I; b, tarsus IV. c, trichobothria, metatarsus IV, dorsal. d,e, tarsus I: d, tarsal organ; e, distal bothrium. f, setae, dorsal abdomen. g,h, metatarsus IV and calamistrum, dorsal. i,j, leg setae, ventral: i, metatarsus; j, tarsus.

with retrodorsal bristle; retrodorsal patellar apophysis usually absent or rudimentary but sometimes well developed; spatulate white hairs present or absent. (Figs. $5 e, g, 1,6 f)$. Cymbium broad with short, broadly conical apex. Tegulum broad with a strong, prolaterally directed, basal tegular bulge. Tegulum and conductor ridged and moderately sclerotised. "S"-shaped, falciform conductor arises from the middle and prolateral tegulum; broadest basally, wrapping across the entire width of the bulb; marginally grooved and distally tapering (Fig. 5d,e,l). Embolus an elongate, sinuous rod, tapering near apex; arising prodorsally from the base of the tegulum, it curves ventrad over the tegular bulge and follows an "S" shaped course with the conductor across the ventral bulb. Median apophysis subapical, membraneous and spoon shaped, directed apically to retrolaterally (Fig. 6d-f). Epigynum with a deep, central fossa bounded posteriorly by a sclerotised transverse ridge of variable width; lateral teeth present, just posterolateral to transverse ridge (Figs. 5c, 7d). Internal genitalia with two wide, membraneous copulatory ducts leading to the paired spermathecae. Each spermatheca consists of a glandulate central lobe connected via a sclerotised interlobar duct of variable length to a small, sometimes indistinct, distal lobe (Figs. 5f, 6g). Spiracle opening anterior to and half as wide as cribellum (Fig. 2a,g). Tracheal system with four branched tubes confined to the abdomen. Calamistrum in central half to two-thirds of metatarsus IV in females, weakly developed in males. 
Spinning organs (Fig. 2a-j, female). Cribellum entire or divided (Fig. 2f,j); spinning field(s) narrow; divided fields well separated (by about one fifth of a field width); males with non-functional, undivided cribellar plate, about three quarters width of functional cribellum. Spinnerets short; ALS broad, 2-segmented, each bordered anteriorly by a row of thick plumose hairs; PMS shortest, 1-segmented; PLS 2segmented, as long as ALS with a coniform terminal segment. In female, ALS with 2 adjacent major ampullate gland spigots medially and about 35 piriform spigots; PMS with 1 minor ampullate, 1 cylindrical and 8 aciniform spigots, plus 4 fused paracribellar bases each with 5-14 spigots; PLS with c. 12 spigots, identified as 8 aciniform, 1-2 cylindrical, 1 modified PLS spigot (base truncate apically) with 2 associated paracribellar spigots, the base of the proximal spigot being partially or completely fused with the modified PLS spigot base (Fig. 2a-e,g-i).

Included species. Phryganoporus candidus (L. Koch), Phryganoporus vandiemeni (Gray) n.comb., P. nigrinus Simon, P. davidleei $\mathrm{n}$. sp., P. melanopygus n.sp.

Notes on synonymies. Phryganoporus Simon is revalidated and removed from its synonymy with Badumna Thorell (Gray, 1983). Phryganoporus nigrinus Simon is removed from synonymy with $B$. candida (L. Koch). Phryganoporus gausapatus (Simon) is returned to synonymy with $P$. candida. Phryganoporus g. gausapatus Simon and $P$. tubicola Simon are retained in synonymy with $P$. candida (L. Koch).

Distribution. Australia and Norfolk Island.

Subgroups. Within Phryganoporus, two subgroups are recognised.

- P. candidus-vandiemeni: Cribellum divided (Fig. 2f). Basal segments of modified PLS spigot and proximal paracribellar spigot joined proximally (Fig. 2e). Calamistrum occupies central half to three-fifths of metatarsus IV (0.48-0.57:1), retrodorsal glabrous zone narrow, distal end of calamistrum usually delimited by a retrodorsal, subdistal spine (Fig. 1g). Transverse epigynal ridge usually broad, lateral teeth broadly pointed (Fig. $5 \mathrm{c}, \mathrm{h}, \mathrm{j})$. Interlobar spermathecal ducts short, curving laterally to the distal spermathecal lobe (Fig. 5f,i,k). Male palp with central limb of the S-shaped embolus/conductor with a strong diagonal slope so that the upper section of the "S" is much smaller than the lower section (Fig. 5d). Patellar apophysis present, vestigial or absent (Fig. 5e,g,l).

- P. davidleei-nigrinus-melanopygus: Cribellum entire (Fig. 2j). Basal segments of modified PLS spigot and proximal paracribellar spigot completely fused (Fig. 2i). Calamistrum occupies central two-thirds of metatarsus IV (0.60-0.70:1), retrodorsal glabrous zone wide, subdistal delimiting spine absent (Fig. 1h). Transverse epigynal ridge usually slender (Figs. 6h, 7d). Interlobar spermathecal ducts elongate, acutely bent ("elbowed") laterally, doubling back to join the small, rounded distal spermathecal lobes (Fig. 6g). Male palp with the central limb of the S-shaped embolus/conductor horizontally oriented or gently sloping so that the upper section of the " $\mathrm{S}$ " is more than half the size of the lower section (Figs. 6d, 9a). Patellar apophysis absent (Fig. 6f).

\section{Remarks}

A previous electrophoretic study of the "Badumna candida species group" (Colgan \& Gray, 1992) supported the validity of Phryganoporus Simon and the synonymy of $P$. gausapatus with $P$. candidus, as well as recognising several more or less distinct genetic populations. Of these, the two most distinct groups proved to be morphologically distinct and are given specific recognition here ( $P$. davidleei n.sp., $P$. melanopygus $\mathrm{n} . \mathrm{sp}$.). All representatives of $B$. vandiemeni were consistently associated in analyses as a distinct basal or near basal clade within the main candidus group. Consequently, despite its close morphological similarity to $P$. candidus, $P$. vandiemeni is maintained here.

Spinnerets. Simon (1908) recorded the presence of an entire cribellum as a characteristic of his new genus Phryganoporus. This observation may have been based upon the female of $P$. nigrinus which he described at that time- the divided cribella of his Amaurobius gausapata and $P$. gausapata occidentalis females perhaps going unnoticed. Main (1971) noted the presence of a divided cribellum in her concept of Ixeuticus candidus. In fact, as demonstrated here, Phryganoporus possesses both entire and divided cribellum states (Fig. 2f,j). These states are correlated with differences in metatarsus IV and calamistrum structure (Fig. 1g,h). Given that the immediate outgroups of Phryganoporus (Badumna, Forsterina) have divided cribellum plates, the direction of change here appears to be from the divided to the entire state. Bond \& Opell (1997) also record the presence of both cribellar states in the American dictynid genus Mallos. In dictynid spiders, including Mallos, the cribellum is typically entire. However, divided cribella characterise representatives of a derived clade in Mallos, within which two sister species show reversion to the entire state. The polarity of cribellar state changes are of considerable phylogenetic interest. The "entire" state seen in the basal araneomorphs (hypochilids and austrochiloids) is considered plesiomorphic for the group, while the basal groups of the Haplogynae and Entelegynae (Filistatidae and Eresoidea) show the derived "divided" state (Griswold $e t$ al., 1999). Ramirez (2000) makes the interesting observation that the early instars of the austrochiloid spider Thaida peculiaris have a "bilobate cribellum". The possibility that this reflects the presence of a divided cribellum state in an ancestral austrochiloid remains open.

The presence of a "modified PLS spigot" and associated paracribellar spigots has been noted in many cribellate entelegyne araneomorphs by Griswold et al. (1999). Griswold (1990) noted that the large modified PLS spigot has a cylindrical but apically tapered base and a cylindrical distal section. In Phryganoporus the base is cylindrical and widely truncate apically, with a distal section that is more or less cylindrical in $P$. candidus and weakly clavate in $P$. nigrinus. Additionally the bases of the modified PLS spigot and the proximal paracribellar spigot are basally fused in $P$. candidus and completely fused in $P$. nigrinus. The pattern seen in P. candidus is also present in Badumna spp. The fusion of these spigots, as well as their proximity, indicates a close functional relationship.

Relationships. Phryganoporus is most closely associated with the Badumna-Forsterina ("badumnine") generic group which is characterised by the sinuous ("S or E-shaped") course of the conductor and embolus across the bulb; and 

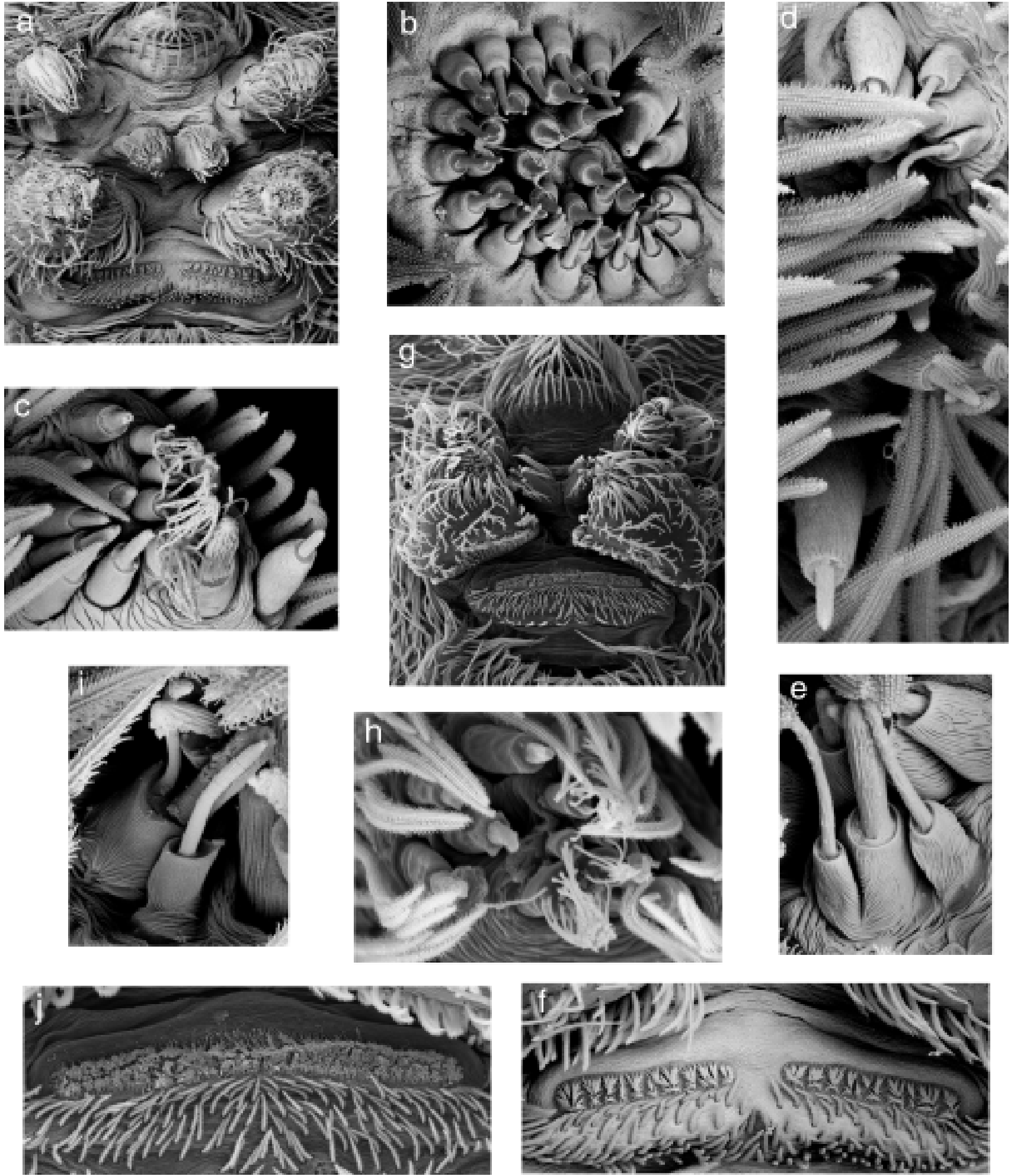

Figure 2. a-j, spinnerets (female). a-f, Phryganoporus candidus: a, spinneret field; b, ALS; c, PMS; d, PLS; e, modified PLS and paracribellar spigots; $\mathrm{f}$, cribellum. g-j, Phryganoporus nigrinus: g, spinneret field; h, PMS; i, modified PLS and paracribellar spigots; $\mathrm{j}$, cribellum.

less closely with the Namandia-Matachia ("namandiine") generic group, which shares with the previous group the "spoon-shaped" membranous median apophysis (or derivative hook-like form or absent), the plate-like (or derivative form) apicoventral RTA and the almost universal presence of epigynal teeth. Morphological trends in Namandia support the derivation of the sinuous embolus/conductor conformation typical of the "badumnine" group from the circular to semicircularfalciform conformation typical of the "namandiine" group. The PMS of genera representing these generic groups differ considerably. In Phryganoporus, Badumna and Forsterina the paracribellar spigot bases are fused while in Namandia, Matachia and Paramatachia they are free, the presumed plesiomorphic condition. 
Like Matachia, many New Zealand genera placed in the Desinae of Forster (1970) and the Amphinectidae of Forster \& Wilton (1973) can be associated with these generic groups. For example, almost all of Forster's desine genera, exemplified by Matachia, Goyenia, Panoa and Manawa, can be related to the "namandiine" group on the basis of palpal (and spinneret) conformation. Of the New Zealand amphinectids, Reinga fits within the "badumnine" group near Forsterina (E-shaped embolus with retrolateral origin) while Mamoea also has "badumnine" affinities (S-shaped embolus with prolateral origin). "Amphinectid-desine" associations have also been suggested by Griswold et al. (1999) and Davies \& Lambkin (2000).

The placement of Phryganoporus here with the Desidae remains provisional. Badumna (syn. Ixeuticus) has been variously associated with the Desidae or Desinae by Lehtinen (1967), Forster (1970) and Griswold et al. (1999). The nominal genus, Desis, is a highly derived taxon whose relationships are unclear. Some of the distinctive characters of Desis include the greatly increased size of the spigot field on the posterior median spinnerets, a double row of trichobothria on the tarsi, apically tapered maxillae, absence of a serrula. Others, like the strongly porrect jaws (also seen in "namandiine" groups and more widely) and reduction of leg spines could be simply specialisations related to living and feeding in an intertidal habitat. The general morphology of the male palp is rather similar to the "namandiine" pattern noted above, but the presence of a "secondary tegular process" is an important difference. Davies (1998) has suggested that this could represent the "primary" conductor (sensu Lehtinen, 1967) whose function has been taken over by the larger falciform "secondary" conductor. This raises doubts over the homology of the functional conductors in Desis and the "badumnine" and "namandiine" groups. All of this suggests that these groups cannot be readily associated with Desis or the Desinae.

\section{Key to species of Phryganoporus}

\section{Females}

1 Cribellum divided. Calamistrum about half as long as metatarsus. Interlobar spermathecal ducts relatively short (observed by depressing the posterior margin of the epigastric groove)

Cribellum entire. Calamistrum about three-quarters length of metatarsus. Interlobar spermathecal ducts elongate and acutely elbowed laterally

2 Distal spermathecal lobes longitudinally ovoid; distal end of interlobar duct bends sharply anterolaterally to join distal lobe. In solitary webs. Tasmania and far southeast coastal mainland .

_ Distal spermathecal lobes usually rounded. Distal end of interlobar duct curves laterally to join distal spermathecal lobe. In solitary or communal webs. Mainland Australia .....

3 Abdominal chevrons not fused posteriorly, sometimes indistinct

Abdominal chevrons fused posteriorly forming a dark patch on the end of the abdomen

4 Body narrow, more or less cylindrical in shape. Abdomen with 23 chevrons and a dark brown, dorsal patch on end of abdomen (eastern populations may be less strongly pigmented) P. nigrinus Body less cylindrical. Abdomen with 1-2 chevrons, posterior third dark brown

\section{Males}

1 Central limb of of S-shaped embolus/conductor strongly angled, diagonal

Central limb of S-shaped embolus/conductor horizontal or only weakly angled

2 Male palp with patellar apophysis and associated white spatulate hairs. In solitary webs. Tasmania and far southeast coastal mainland

Male palp usually without obvious patellar apophysis. In solitary or communal webs. Mainland Australia 
3 Abdominal chevrons not fused posteriorly, sometimes indistinct P. davidleei Abdominal chevrons fused posteriorly forming a dark patch on the end of the abdomen 4

4 Central limb of S-shaped conductor horizontal. Body narrow, more or less cylindrical in shape. Abdomen with 2-3 chevrons and a dark brown, dorsal patch on end of abdomen (eastern populations may be less strongly pigmented)

P. nigrinus

_ Central limb of S-shaped conductor weakly angled. Body less cylindrical. Abdomen with 1-2 chevrons, posterior third dark brown

P. melanopygus

\section{Phryganoporus candidus (L. Koch)}

Figs. 1a,g, 2a-f, 3a,b, 4a, 5a-i

Amaurobius candidus L. Koch, 1872: 333, pl.25, fig. 6. Holotype female from Bowen, Queensland, Australia (ZMH, Araneae type catalogue no. 11, Museum Godeffroy, Hamburg, catalogue no. 7852-examined).

Amaurobius gausapatus Simon, 1906: 295.

Phryganoporus gausapatus.-Simon, 1908: 367. Lehtinen, 1967: 259, fig. 102.

Phryganoporus gausapatus occidentalis Simon, 1908: 367. Main \& Harvey, 1992: 708.

Phryganoporus tubicola Simon, 1908: 367, fig. 1. Lehtinen, 1967: 259, fig. 109. Main \& Harvey, 1992: 708.

Ixeuticus candidus.-Roewer, 1954: 1388. Hickman, 1967: 33, fig. 50, 51 [ = P. vandiemeni (Gray)]. Leech, 1972: 26. Main, 1971: 119. (Not Dondale, 1966: 1188, fig. 9a-e [= Badumna sp.]).

Phryganoporus candidus.-Lehtinen, 1967: 259.

Badumna gausapata.-Gray, 1983: 254, fig. 12-18. Platnick, 1989: 408; 1997: 629.

Badumna candida.-Gray, 1983: 250, fig. 1-11. Platnick, 1989: 408; 1993: 536; 1997: 629. Colgan \& Gray, 1992: 86.

Material examined. NEW SOUTH WALES: 4 \% , KS6006, Wee Jasper, on Carey's Rd, $35^{\circ} 07^{\prime} \mathrm{S} 148^{\circ} 41^{\prime} \mathrm{E}, 30$ Oct 1980 , MRG; from solitary webs on dead thistle heads. 19 , KS6088, data as for KS6006. 230 , $19,2 j$, KS6089, $6.5 \mathrm{~km}$ E of Dubbo, 32 $2^{\circ} 14^{\prime} \mathrm{S} 149^{\circ} 10^{\prime} \mathrm{E}, 21$ Aug 1980, MRG \& $\mathrm{CH}$; communal web. 1 ㅇ, $\mathrm{KS} 6936,21 \mathrm{~km}$ E of Parkes, $33^{\circ} 08^{\prime} \mathrm{S} 148^{\circ} 11^{\prime} \mathrm{E}$, 8 Apr 1972, MRG; solitary web. 60, 4 ㅇ, KS6937, Baradna Downs, Wanaaring, $29^{\circ} 42^{\prime} \mathrm{S} 144^{\circ} 09^{\prime} \mathrm{E}, 28 \mathrm{Mar} 1977$; on hop bush. 1 ㅇ, KS6940, Round Hill, $32^{\circ} 59^{\prime}$ S $146^{\circ} 09^{\prime} \mathrm{E}, 1$ Aug 1968, MRG. 1 \% , KS6979, data as for KS6940, 5 Aug 1968; web on spinifex. 1 ㅇ, KS6982, Bourke area, $30^{\circ} 05^{\prime} \mathrm{S} 145^{\circ} 56^{\prime} \mathrm{E} .1$ ㅇ, KS 7852 , Warrumbungle National Park, $31^{\circ} 19^{\prime} \mathrm{S}$ $148^{\circ} 59^{\prime} \mathrm{E}, 1$ Feb 1969 , MRG. 3 \% , KS15958, Mildura, $34^{\circ} 11^{\prime} \mathrm{S} 142^{\circ} 10^{\prime} \mathrm{E}$, 17 Dec 1978, MRG; communal nests on mallee. 21 $0^{\star}, 8$ \% , KS16731, Hay-Grenfell area, $34^{\circ} 24^{\prime} \mathrm{S} 146^{\circ} 46^{\prime} \mathrm{E}, 12$ May 1986, MRG. $1 \delta^{\circ}, 1 \%$,

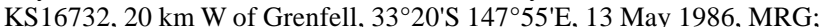
web on eucalypt. $10^{\dagger}, \mathrm{KS} 16733,60 \mathrm{~km}$ E of Hay, $34^{\circ} 29^{\prime} \mathrm{S} 145^{\circ} 29^{\prime} \mathrm{E}, 10$ May 1986, MRG; web on wattle. 10, 1 \% , KS17161, 2 km E of Gunbar, $34^{\circ} 04^{\prime} \mathrm{S} 145^{\circ} 25^{\prime} \mathrm{E}, 24 \mathrm{Apr} 1987$, MRG; 26 spiders in communal nest on

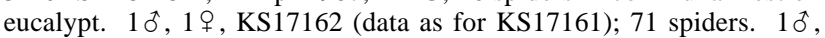
1 ㅇ, KS17163 (data as for KS17161); 46 spiders. $20^{7}, 1$ 우, KS17164 (data as for KS17161); 100 spiders. 19 , KS17452, Goonoo State Forest, NW corner near "Ultimo" Station, 3203'S 148 ${ }^{\circ} 55^{\prime}$ E, 17 Sep 1987, CH

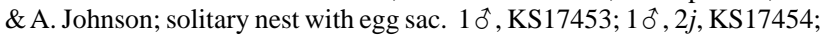
19 , KS17455; Goonoo State Forest, NE corner N of junction of Breelong \& Denmire Rds, $32^{\circ} 03^{\prime} \mathrm{S} 148^{\circ} 55^{\prime} \mathrm{E}, 18 \mathrm{Sep} 1987, \mathrm{CH} \&$ A. Johnson. 1 \% KS17457, Goonoo State Forest, SW corner, junction of Freemans \& Western Boundary Rds, $32^{\circ} 03^{\prime} \mathrm{S} 148^{\circ} 55^{\prime} \mathrm{E}, 18 \mathrm{Sep} 1987, \mathrm{CH} \&$ A. Johnson. $74 j$, KS17460, south of Neville on Kentucky Rd, "Springerlee" Station gate, $33^{\circ} 45^{\prime} \mathrm{S} 149^{\circ} 12^{\prime} \mathrm{E}, 19 \mathrm{Sep} 1987$, CH \& A. Johnson. 1 ㅇ, KS17822, Lake Munmorah State Recreation Reserve, Geebung, $33^{\circ} 13^{\prime} \mathrm{S} 151^{\circ} 34^{\prime} \mathrm{E}$, 30 Oct 1987, MRG; from communal web (used for electrophoretic study). 1 ㅇ, KS32719, Newtown, $33^{\circ} 37^{\prime} \mathrm{S} 150^{\circ} 48^{\prime} \mathrm{E}$, March 1928, F. Plant. 19 ,

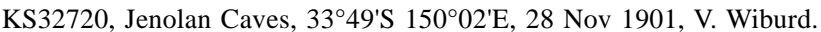

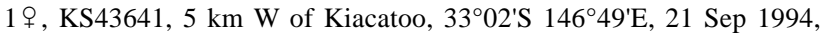
MRG; nest with juveniles on riparian eucalypt. 1 \%, KS43642, $7 \mathrm{~km} \mathrm{~W}$ of Parkes, $33^{\circ} 06^{\prime} \mathrm{S} 148^{\circ} 07^{\prime} \mathrm{E}, 21$ Sep 1994, MRG; solitary nest on Callitris.
1 ㅇ, 1j, KS43643, $10 \mathrm{~km}$ NW of Wagga Wagga on Coolamon Road, $35^{\circ} 03^{\prime} \mathrm{S} 147^{\circ} 22^{\prime} \mathrm{E}, 23 \mathrm{Sep} 1994$, MRG. 1 \% , KS43661, $36 \mathrm{~km} \mathrm{SW}$ of Bingara on Narrabri Rd, Glacial Area Historical Site, $30^{\circ} 03^{\prime} \mathrm{S} 150^{\circ} 17^{\prime} \mathrm{E}$,

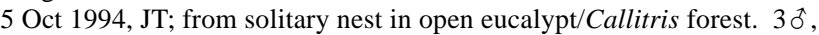
1 , KS43662, data as for KS43661; in communal nest. 2 9 , N19922023 (SAM), Broken Hill, $31^{\circ} 58^{\prime}$ 'S 141 ${ }^{\circ} 27^{\prime} \mathrm{E}, 12$ Jul 1980, DH. $10^{\circ}, 2$, N1992182-4 (SAM), Menindee Lakes, 32 ${ }^{\circ} 20^{\prime}$ S $142^{\circ} 20 '$ 'E, 11 Apr 1981, DH. Australian CAPITAL Territory: 19 , KS6087, Black Mountain, Canberra, $35^{\circ} 17^{\prime} \mathrm{S} 149^{\circ} 13^{\prime} \mathrm{E}, 1$ Oct 1980 , MRG; solitary web, egg sac with 29 eggs. 19 , KS6090, Black Mountain, Canberra, $35^{\circ} 17^{\prime} \mathrm{S} 149^{\circ} 13^{\prime} \mathrm{E}$, 1 Nov 1980, MRG; from communal web. 49 , KS6086, data as for KS6090; from communal web, 4 egg sacs with 13, 49, 22 and 39 eggs. 5 o, KS6942, Canberra, $35^{\circ} 17^{\prime} \mathrm{S} 149^{\circ} 13^{\prime} \mathrm{E}$, 24 May 1965, M.S. Upton; collected from ANIC light trap. $19,2 j$, KS17153, Black Mountain, Canberra, $35^{\circ} 16^{\prime} \mathrm{S} 149^{\circ} 06^{\prime} \mathrm{E}, 25 \mathrm{Apr} 1987$, MRG; $48 j$ in nest. VICTORIA: $40{ }^{\circ}, 23 \stackrel{9}{9}, 8 j$, KS $17177,18 \mathrm{~km} \mathrm{~W}$ of Mildura, $34^{\circ} 14^{\prime} \mathrm{S} 141^{\circ} 58^{\prime} \mathrm{E}, 24 \mathrm{Apr}$ 1987, MRG; part of large communal nest of 558 individuals on mallee eucalypt by roadside. $10 \AA, 3 q, 12 j$, KS17154 (data as for KS17177); nest with 150 specimens. $2 \hat{\delta}, 2$ q, $4 j$, KS17155 (data as for KS17177); 106 specimens in nest. $8 \hat{0}, 9 q, 8 j, \mathrm{KS} 17156$ (data as for KS17177); 172 specimens in nest. 16 \% $\mathrm{KS} 31601-4,17 \mathrm{~km}$ NE of Euroa, 36 $39^{\circ} \mathrm{S}$ $145^{\circ} 43^{\prime} \mathrm{E}, 25$ Oct 1990, MRG; from solitary nests on shrubs. 6 우, $\mathrm{KS} 34441$, data as for KS31601-4. $29, \mathrm{KS} 31605,2 \mathrm{~km}$ E of Rushworth, on Murchison road, $36^{\circ} 36$ 'S $145^{\circ} 02^{\prime} \mathrm{E}, 13 \mathrm{Feb} 1991$, CH \& JT; web on spiky shrub. $10^{\top}$, (NMV), $16.2 \mathrm{~km} \mathrm{SE}$ of Murrayville, $35^{\circ} 22^{\prime} \mathrm{S} 141^{\circ} 19^{\prime} \mathrm{E}$,

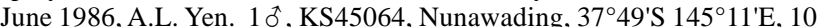
Jun 1956, A. Neboiss. 2\%, N1992192-3 (SAM), Harriers Swamp Holey Plains State Park, $38^{\circ} 14^{\prime}$ S $146^{\circ} 56^{\prime} \mathrm{E}, 16$ Jun 1989, DH. $10^{\star}$, N1992204 (SAM), Red Cliffs, 1978, DH. NORTHERN TERRITORY: 2 q, KS55828, Watarrka National Park, King’s Canyon, $24^{\circ} 15^{\prime} \mathrm{S} 131^{\circ} 31^{\prime} \mathrm{E}, 16$ May 1999 , MRG, GAM \& HS. 19, KS55829, Uluru National Park, Ranger accommodation area, $25^{\circ} 21^{\prime} 16^{\prime \prime S} 131^{\circ} 04^{\prime} 11^{\prime E}$, 14 May 1999, MRG, GAM \& HS; on prickly Acacia. 1 9 , KS55830, Watarrka National Park, Kathleen Springs, 2420'38"S 131 40'54"E, 15 May 1999, MRG, GAM $\&$ HS. SOUTH AUSTRALIA: $10^{\top}, 8+9,10 j$, KS8663, Kimba, Eyre Peninsula, $33^{\circ} 08^{\prime} \mathrm{S} 136^{\circ} 25^{\prime} \mathrm{E}, 30$ Mar 1978, B.Y. Main. 9ô, 6 우 $1 j$, KS16925, Acraman Ck, Eyre Peninsula, track E of Smoky Bay, $32^{\circ} 23^{\prime} \mathrm{S} 133^{\circ} 56^{\prime} \mathrm{E}$, 7 May 1986, B.Y. Main; communal nest on mallee eucalypt. $10^{\dagger}, 1$ 웅, $\mathrm{KS} 17157,33 \mathrm{~km}$ W of Iron Knob, 32 $2^{\circ} 07 ' \mathrm{~S} 136^{\circ} 50^{\prime} \mathrm{E}, 24 \mathrm{Apr} 1987$, MRG; 41 specimens in communal web on mallee eucalypt. $20^{\star}, 1$ \% , KS17158 (data as for KS17157); 37 specimens in communal web. $20,1 \%$, KS17159 (data as for KS17157). $1 \hat{0}, 29,3 j, \mathrm{KS} 17160$ (data as for KS17157); 22 specimens in communal web. $19, j$, KS34473, Nullarbor Plain nr Catacombs Cave (5N), 31 ${ }^{\circ} 08^{\prime} \mathrm{S} 130^{\circ} 37^{\prime} \mathrm{E}, 10 \mathrm{Jul}$ 1991, MRG; solitary web on Acacia. 5 + , 3j, KS34474, data as for KS34473; solitary tubular retreat web on samphire. $19, \mathrm{KS} 34475$, Nullarbor Plain, grid ref 663.8 6527, $11 \mathrm{Jul}$ 1991, MRG; nest on Acacia shrub in cave doline. 1 웅, KS55827, Orroroo, at Giant Red Gum, 32²4'S $138^{\circ} 37^{\prime} \mathrm{E}, 10$ May 1999, MRG, GAM \& HMS. 우, $j$, N1991281 (SAM), $4 \mathrm{~km} \mathrm{SW}$ of Emu, $28^{\circ} 35^{\prime} \mathrm{S} 132^{\circ} 10^{\prime} \mathrm{E}, 6$ Oct 1976, DCL; communal nest on sandhill Acacia. $19, j, \mathrm{~N} 1991282(\mathrm{SAM}), 13 \mathrm{~km}$ E of Ooldea, $30^{\circ} 28^{\prime} \mathrm{S} 131^{\circ} 58^{\prime} \mathrm{E}, 30 \mathrm{Sep}$ 1988, DH; on low mallee. 6 ? , N1991283-8 (SAM), $13 \mathrm{~km} \mathrm{E} \mathrm{of} \mathrm{Ooldea,}$ $30^{\circ} 28^{\prime} \mathrm{S} 131^{\circ} 58^{\prime} \mathrm{E}, 30 \mathrm{Sep} 1988$, DH. 1 \%, N1991289 (SAM), Lake Meramangye, near SW corner, $28^{\circ} 29^{\prime} \mathrm{S} 132^{\circ} 08^{\prime} \mathrm{E}, 6$ Oct 1976, DCL; in communal web on Acacia. 19 , N1991290 (SAM), $5 \mathrm{~km}$ E of Gibraltar Rocks, $30^{\circ} 26^{\prime} \mathrm{S} 134^{\circ} 31^{\prime} \mathrm{E}, 2$ Oct 1976, DCL; on Melaleuca. 1 우, N1991291 (SAM), Yardea Homestead, 32 ${ }^{\circ} 23^{\prime} \mathrm{S} 135^{\circ} 31^{\prime} \mathrm{E}, 25$ Sep 1988, DH. 1 ㅇ, N1991292 (SAM), Gawler Ranges, 343ㅇ' $138^{\circ} 45^{\prime} \mathrm{E}$, October 1985, NPWS Survey. 1q, $j$, N1991293 (SAM), Kolay Hut, 32³3'S $135^{\circ} 36^{\prime} \mathrm{E}, 9$ Dec 1989, DH. 1 \%, N1991294-5 (SAM), Kulliparu Conservation Park, SW corner (NW cnr Courtabie lease), 330ㅗ'S 


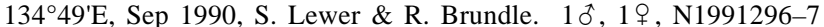
(SAM), Mt Wedge, $1 \mathrm{~km} \mathrm{NW}$ of summit, 33⒉' $135^{\circ} 09^{\prime} \mathrm{E}, 31$ Mar 1987,

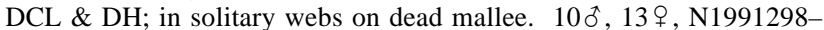

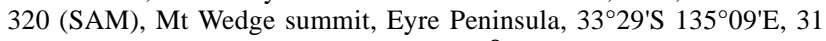
Mar 1987; communal nest on mallee. 3 $9,54 j$, N1991321-3 (SAM), data as for N1991298-320. $10 \%, 5 j$, N1991324-33 (SAM), Carrappee Hill Conservation Park, 2 Apr 1987, DCL \& DH; in communal web on mallee. $19,8 j$, N1991334 (SAM), Hinks Conservation Park SE Boundary, 25 Mar 1987, DCL \& DH; in communal web. 1 ơ, N1991335 (SAM), Lake Gilles Conservation Park, 32 $38^{\circ}$ 'S $136^{\circ} 53^{\prime} \mathrm{E}, 2$ Apr 1987, DCL \& DH; solitary web on dead mallee. 1 \% , N1991336 (SAM), Pinkawillinie Conservation Park, $33^{\circ} 07^{\prime} \mathrm{S} 136^{\circ} 00^{\prime} \mathrm{E}, 18-19$ May 1990, DH. $10^{\star}, 1+, j$, N1991337-9 (SAM), Nacooma Station $35 \mathrm{~km}$ N of Port Augusta, 32 ${ }^{\circ} 13^{\prime} \mathrm{S}$ $137^{\circ} 52^{\prime} \mathrm{E}, 10$ May 1982 , G. Traice; web on saltbush. $1 \delta^{\widehat{t}}, 3+, 1 j$, N1991340-4 (SAM), 2-3 km W of Lake Frome, 3044'S 139 $46^{\circ}$ E, 10 May 1989, J.A. Forrest \& DCL. 1 \% , N1991345 (SAM), Melrose camping area, 32 ${ }^{\circ} 50^{\prime} \mathrm{S} 138^{\circ} 11^{\prime} \mathrm{E}, 18 \mathrm{Apr} 1987$, DH. 30, 1 \% , 6j, N1991346-9 (SAM), Bald Hill Beach near Port Wakefield, $34^{\circ} 11^{\prime} \mathrm{S} 138^{\circ} 09^{\prime} \mathrm{E}, 11 \mathrm{Feb}$ 1989, B. McMahon. $19, j$, N1991352-5 (SAM), 4 km from FerriesMcDonald National Park on Strathally Rd, $35^{\circ} 13^{\prime} \mathrm{S} 139^{\circ} 09^{\prime} \mathrm{E}, 24 \mathrm{Nov}$ 1980, G. Coombs; communal nest on eucalypt. 7\%, N1992129-136 (SAM), S of Middleback Station, 32 ${ }^{\circ} 57^{\prime}$ S $137^{\circ} 23^{\prime}$ E, June 1984, BG; "web cases" in solitary webs on Callitris, Lignum, Scaevola, myall, Lycium. 10 , N1992200 (SAM), Middleback Station Vagrantin Shearer's Kitchen, $32^{\circ} 57^{\prime} \mathrm{S} 137^{\circ} 23^{\prime} \mathrm{E}$, June 1984, BG. 1 ô $^{\hat{2}}$ N1992201 (SAM), South

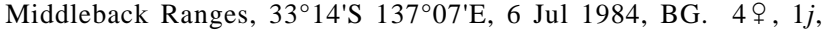
N1992151-5 (SAM), Hambridge Conservation Park, 332ㄱ'S $135^{\circ} 56^{\prime} \mathrm{E}$, April 1984, BG. 14 , N1992157-70 (SAM), Nappyalla, 35²6'S $139^{\circ} 07^{\prime} \mathrm{E}$, October 1991, J. Eckert. 19 , N1992171 (SAM), $13 \mathrm{~km} \mathrm{~N}$ of Keilira Station, $36^{\circ} 37^{\prime} \mathrm{S} 140^{\circ} 11^{\prime} \mathrm{E}, 10$ May 1968, DH. 1 \% , N1992185 (SAM), Appreentinna Ck, $27^{\circ} 48^{\prime} \mathrm{S} 134^{\circ} 09^{\prime} \mathrm{E}, 21$ May 1981, DH. 1 \% , N1992186-9 (SAM), Port Augusta, 32 ${ }^{\circ} 30^{\prime} \mathrm{S} 137^{\circ} 46^{\prime} \mathrm{E}, 23-25$ Jan 1987, $\mathrm{DH}$; coastal sand dune habitat. QUEENSLAND: 30 , 4 \% , $2 j$, KS42443, 45 $\mathrm{km} \mathrm{S}$ of Charters Towers, $20^{\circ} 29^{\prime} \mathrm{S} 146^{\circ} 14^{\prime} \mathrm{E}, 24$ Apr 1994, M. Moulds \& JT; from communal nest in Acacia tree. 19, KS43576, Forty Mile Scrub $1 \mathrm{~km}$ from Mt Surprise turnoff, $18^{\circ} 05^{\prime} \mathrm{S} 144^{\circ} 53^{\prime} \mathrm{E}, 12 \mathrm{Apr} 1994$, JT, M. Moulds, F. MacKillop; communal web on spiky dead bush. 2 ㅇ, KS43646, McLeod R. crossing beside highway, $16^{\circ} 30^{\prime}$ S $145^{\circ} 00^{\prime} \mathrm{E}, 16 \mathrm{Apr} 1994$, JT, M. Moulds, F. Mackillop. 19, KS43647, Annan R. crossing beside highway, $15^{\circ} 41^{\prime} \mathrm{S} 145^{\circ} 12^{\prime} \mathrm{E}, 16$ Apr 1994, JT, M. Moulds, F. Mackillop.

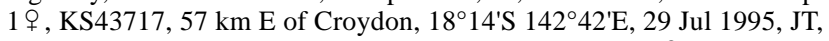
M. Moulds; communal web in bottlebrush shrub. 19, KS43930,

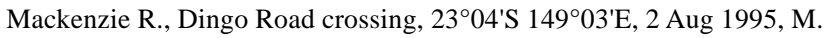
Moulds, M. Tio. 20 $, 10 \%, 1 j$, KS43974, Mackenzie R., Dingo Rd Crossing, $23^{\circ} 04^{\prime} \mathrm{S} 149^{\circ} 03^{\prime} \mathrm{E}, 2$ Aug 1995, M. Moulds, M. Tio. 1 \% , KS43975, between Carnarvon Gorge turn-off \& Rolleston, on Highway, $24^{\circ} 53^{\prime} \mathrm{S} 148^{\circ} 31^{\prime} \mathrm{E}, 8 \mathrm{Jul} 1995$, JT, M. Tio, S. Cowan; solitary web, on

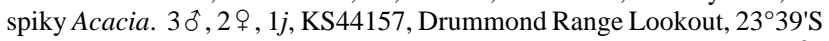
$147^{\circ} 12^{\prime} \mathrm{E}, 8$ Jul 1995 , JT, M. Tio, S. Cowan; communal web. 2 ㅇ, $\mathrm{KS} 44160,95 \mathrm{~km} \mathrm{~S}$ of Rolleston on highway to Injune, $25^{\circ} 14^{\prime} \mathrm{S} 148^{\circ} 37^{\prime} \mathrm{E}$, 6 Jul 1995, JT, M. Tio, S. Cowan; web on spiky bushes. 2 9, KS44162, $80 \mathrm{~km} \mathrm{NW}$ of Winton on (station) road to Cloncurry, $21^{\circ} 56^{\prime} \mathrm{S} 142^{\circ} 32^{\prime} \mathrm{E}$, 10 Jul 1995, JT, M. Tio, S. Cowan; in communal web. 2 \%, KS44163, NW of Winton on highway, $22^{\circ} 12^{\prime} \mathrm{S} 142^{\circ} 53^{\prime} \mathrm{E}, 10 \mathrm{Jul} 1995$, JT, M. Tio, S. Cowan; communal web. 19, KS44165, Chillagoe National Park outside Royal Arch Cave, $17^{\circ} 11^{\prime}$ S $144^{\circ} 29^{\prime}$ E, 16 Jul 1995, JT, M. Tio, S. Cowan; in communal web. 19 , KS44166, Undara National Park, $18^{\circ} 12^{\prime}$ S $144^{\circ} 34^{\prime} \mathrm{E}, 29$ Jul 1995, JT, M. Tio, S. Cowan; communal web. 19 , KS44171, $19 \mathrm{~km} \mathrm{~S}$ of Burke \& Wills Roadhouse on highway to Normanton, $19^{\circ} 20^{\prime} \mathrm{S} 140^{\circ} 15^{\prime} \mathrm{E}, 12 \mathrm{Jul} 1995$, JT; in communal webs. 1 ㅇ, 2j, KS44172, 62 km W of Chillagoe, Burke Development Rd, 16 ${ }^{\circ} 44^{\prime} \mathrm{S}$ $144^{\circ} 05^{\prime} \mathrm{E}, 15$ Jul 1995, JT, S. Cowan; communal web. 1 9 , 3j, KS44173, $5 \mathrm{~km} \mathrm{~S}$ of Normanton, $17^{\circ} 46^{\prime} \mathrm{S} 141^{\circ} 01^{\prime} \mathrm{E}, 12 \mathrm{Jul} 1995 \mathrm{JT}$, S. Cowan; from communal web. $1 \hat{0}, \mathrm{KS} 44174,30 \mathrm{~km} \mathrm{~S}$ of St. George, beside highway to Mungindi, $28^{\circ} 12^{\prime} \mathrm{S} 148^{\circ} 44^{\prime} \mathrm{E}$, 02 Aug 1995 , JT; communal web in small Acacia. $19,3 j$, KS44190, $95 \mathrm{~km} \mathrm{~S}$ of Injune on highway to Rolleston, $25^{\circ} 14^{\prime} \mathrm{S} 148^{\circ} 37^{\prime} \mathrm{E}, 06 \mathrm{Jul} 1995$, JT, M. Tio, S. Cowan; web on spiky bushes. 1 ㅇ, KS44191, $20 \mathrm{~km}$ N of Cloncurry, 20³3'S 140²5'E, 11 Jul 1995, JT; from communal web on dead Acacia tree. $19, \mathrm{KS} 44192, \mathrm{~N}$ of Hann R. on highway to Coen, $15^{\circ} 02^{\prime} \mathrm{S} 143^{\circ} 40^{\prime} \mathrm{E}, 18 \mathrm{Jul} 1995$, JT, J. Olive. $3 q, 2 j$, KS44193, Belmore Ck, $10 \mathrm{~km} \mathrm{~W}$ of Croydon, $18^{\circ} 02^{\prime} \mathrm{S} 141^{\circ} 58^{\prime} \mathrm{E}, 29 \mathrm{Jul}$ 1995, JT, M. Moulds; communal webs on Acacia. 1 ㅇ, 1j, KS44194, 25 $\mathrm{km}$ NE of the Lynd, $18^{\circ} 42^{\prime} \mathrm{S} 144^{\circ} 40^{\prime} \mathrm{E}, 30 \mathrm{Jul} 1995$, JT; on Acacia. 19 ,

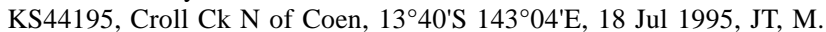
Moulds, M. Tio, F. MacKillop, J. Olive; in communal web. 1 万ิ, KS44843, on track to Moss Garden, $25^{\circ} 03^{\prime} \mathrm{S} 148^{\circ} 12^{\prime} \mathrm{E}, 07 \mathrm{Jul} 1995$, JT, M. Moulds; solitary web on dead thistle. $80^{2}, 7 q, 6 j$, KS45063, JCU Campus, Townsville, $19^{\circ} 16^{\prime} \mathrm{S} 146^{\circ} 49^{\prime} \mathrm{E}, 1986-89$, M. Downes; from several nests.
$19, \mathrm{KS} 45727$, Silver Valley near Herberton, $17^{\circ} 20^{\prime} \mathrm{S} 145^{\circ} 17^{\prime} \mathrm{E}, 24$ Sep 1950, J.S. Brookes. 3 0 , 2 \% , S141 (QM), Girraween National Park area, $28^{\circ} 50^{\prime} \mathrm{S} 151^{\circ} 55^{\prime} \mathrm{E}, 7 \mathrm{Apr} 1974$, R.J. Raven; on heath. $40^{\hat{2}}, 2$ ㅇ, 23j, S144 (QM), Southwood, $30 \mathrm{~km} \mathrm{~W}$ of Moonie, 27 ${ }^{\circ} 46^{\prime} \mathrm{S} 150^{\circ} 04^{\prime} \mathrm{E}, 24$ Aug 1973, R.J. Raven; communal web 35-40 cm diameter on Hakea. 4ðे, $19,3 j$, S15942 (QM), Kenniff Cave area Mt Moffatt, $25^{\circ} 01^{\prime} \mathrm{S} 147^{\circ} 57^{\prime} \mathrm{E}, 27$ Sep 1986, M. Bennie. 10 ㅇ, $1 j \mathrm{~S} 20199$ (QM), Chillagoe, $17^{\circ} 09^{\prime} \mathrm{S} 144^{\circ} 31^{\prime} \mathrm{E}$,

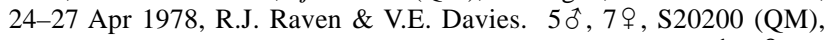
Eureka Ck, $17^{\circ} 11^{\prime} \mathrm{S} 145^{\circ} 01^{\prime} \mathrm{E}, 11 \mathrm{Feb} 1972$, N.C. Coleman. $1 \mathrm{o}^{\wedge}, 4 \stackrel{+}{ }, 1 j$, S20201 (QM), Wolfram, $17^{\circ} 05^{\prime} \mathrm{S} 144^{\circ} 57^{\prime} \mathrm{E}, 13 \mathrm{Feb} 1972$, N.C. Coleman. $10^{\circ}, 3$ 오, S20202, Mt Garnet, $17^{\circ} 41^{\prime} \mathrm{S} 145^{\circ} 06$ 'E, 24 Feb 1972. 1 우, (QM), Kroombit Tops, $24^{\circ} 22^{\prime} \mathrm{S} 151^{\circ} 01^{\prime} \mathrm{E}$. 1 \% , (QM), Endfield (Mulga site), $23^{\circ} 46^{\prime} \mathrm{S} 151^{\circ} 16^{\prime} \mathrm{E} .1 \%$, (QM), Moreton Island near Trench, $27^{\circ} 11^{\prime} \mathrm{S}$ $153^{\circ} 24^{\prime} \mathrm{E} .19$, (QM), Glenormiston, $22^{\circ} 55^{\prime} \mathrm{S} 138^{\circ} 48^{\prime} \mathrm{E}$. 1 ㅇ, (QM), Mt Garnet, $17^{\circ} 41^{\prime} \mathrm{S} 145^{\circ} 07^{\prime} \mathrm{E} .19,2 j$, N1992172 (SAM), near Tiaro, $25^{\circ} 44^{\prime} \mathrm{S}$ $152^{\circ} 35^{\prime} \mathrm{E}, 25$ May 1979, DH. Western Australia: $10^{\dagger}$, Ar.811 (MNHN), syntype of Phryganoporus tubicola Simon (examined), Denham, (Stat. 65). 2j, 11/4279, 4279a (WAM), syntypes of P. tubicola Simon (not seen, other data as for Ar.811). 1j, 11/4269 (WAM), syntype of Phryganoporus gausapatus occidentalis Simon, East Cannington (Stat. 125). 1 ㅇ, KS6938, Torbay Head, $35^{\circ} 08^{\prime} \mathrm{S} 117^{\circ} 38^{\prime} \mathrm{E}, 10$ Oct 1977 , MRG. $10^{\top}, 4$ ㅇ, KS6939, Brookton, $32^{\circ} 22^{\prime} \mathrm{S} 117^{\circ} 00^{\prime} \mathrm{E}, 5$ May 1977, MRG. 1 웅. KS6981, Perth, $31^{\circ} 57^{\prime} \mathrm{S} 115^{\circ} 51^{\prime} \mathrm{E} .19$, KS9986, Dryandra Forest near Narrogin (site 2(22)), 32 $56^{\circ} \mathrm{S} 117^{\circ} 11^{\prime} \mathrm{E}, 29$ Mar 1982, K. Brown. 1 운, KS9987, Dryandra Forest (site 2(15)), data as for KS9986. 10ิ, 1 \%, KS10252, Brookton, $32^{\circ} 22^{\prime} \mathrm{S} 117^{\circ} 00^{\prime} \mathrm{E}, 5$ May 1977 ; on citrus tree. 1 ㅇ. KS17357, Anna Plains Station, $25 \mathrm{~km}$ N of Sandfly Flat, $19^{\circ} 15^{\prime} \mathrm{S} 121^{\circ} 29^{\prime} \mathrm{E}$,

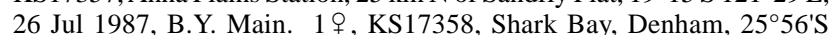
$113^{\circ} 32^{\prime} \mathrm{E}, 5$ Jul 1987, B.Y. Main. 19, 59j, KS17447, Canning Stock Route, west of Well $41,21^{\circ} 34^{\prime} \mathrm{S} 125^{\circ} 51^{\prime} \mathrm{E}, 8$ Aug 1987, AEJ. $22 j$, KS17448, Canning Stock Route, at cairn approx. $20 \mathrm{~km} \mathrm{NE}$ of Well 36, $22^{\circ} 00^{\prime} \mathrm{S} 125^{\circ} 07^{\prime} \mathrm{E}, 6$ Aug 1987, AEJ. 72j, KS17449, Canning Stock Route, track to Well $37,22^{\circ} 09^{\prime} \mathrm{S} 125^{\circ} 19^{\prime} \mathrm{E}, 6$ Aug 1987, AEJ; on Acacia. 37j, KS17450, Canning Stock Route, near Well 31, $22^{\circ} 31^{\prime} \mathrm{S} 124^{\circ} 23^{\prime} \mathrm{E}$, 4 Aug 1987, AEJ; on Acacia. 1 9 , 91/1616 (WAM), Canning Stock Route, Well $6,25^{\circ} 14^{\prime} \mathrm{S} 121^{\circ} 05^{\prime} \mathrm{E}, 22 \mathrm{Jul} 1987$, AEJ; from large nest on Acacia, several spiders seen. $19,91 / 1617$ (WAM), Canning Stock Route, nr Well 23, $23^{\circ} 05^{\prime} \mathrm{S} 123^{\circ} 12^{\prime} \mathrm{E}, 29 \mathrm{Jul}$ 1987, AEJ. 1ㅇ, 91/1618 (WAM), Canning Stock Route, nr Well 23, $23^{\circ} 05^{\prime} \mathrm{S} 123^{\circ} 12^{\prime} \mathrm{E}$, $30 \mathrm{Jul} 1987$, AEJ. $4 j$, KS19007-10, Cape Bernier, Kimberley area, $15^{\circ} 39^{\prime} \mathrm{S} 128^{\circ} 42^{\prime} \mathrm{E}$, 06 Jun 1988, A.R. Main. 1 ㅇ, KS19270, South Hedland, 20²6'S $118^{\circ} 36^{\prime} E, 9$ Aug 1988, P. Filmer-Sankey; ex Acacia translucens, communal nest. 4 , KS23447, Collier Range National Park, $24^{\circ} 32^{\prime} \mathrm{S} 118^{\circ} 50^{\prime} \mathrm{E}$, 14 Jul 1990 , AEJ. 19 , KS 23449 , Mellin Jerrie Well, Kidson Track, $21^{\circ} 01^{\prime} \mathrm{S} 123^{\circ} 20^{\prime} \mathrm{E}$, 28 Jul 1990, AEJ. $j$, AMS (unregistered), Point Spring Nature Reserve, NNE of Kununurra, $15^{\circ} 24^{\prime} 37^{\prime \prime S} 128^{\circ} 52^{\prime} 58^{\prime \prime E}, 29$ May 1999, MRG, GAM $\&$ HMS; communal web. $19, j$, KS55831, Durack R. Crossing, Gibb R. Road, $15^{\circ} 56^{\prime} \mathrm{S} 127^{\circ} 13^{\prime} \mathrm{E}$, 04 Jun 1999, MRG, GAM \& HMS; communal web on foliage. $19, j$, KS55832, data as KS55831. $1{ }^{\star}, 91 / 1611$ (WAM), Belmont International Airport, Perth, along $\mathrm{N}$ fence off Kalamunda Road, $31^{\circ} 57^{\prime} \mathrm{S} 115^{\circ} 51^{\prime} \mathrm{E}, 20 \mathrm{Jan} 1987$, W.F. Humphreys; in tubular web. 1 q , $16 j, 91 / 1620-37$ (WAM), City Beach nr Rifle Range, $31^{\circ} 56^{\prime} \mathrm{S} 115^{\circ} 45^{\prime} \mathrm{E}$, 21 Jun 1973, Mercedes College 3rd yr. 2q, 1j, 91/1639-41 (WAM), Darlington, $31^{\circ} 54^{\prime} \mathrm{S} 116^{\circ} 04^{\prime} \mathrm{E}$, May 1975-Mar 1976, G.H. Lowe. $10^{\circ}$, $19,91 / 1642-3$ (WAM), Darlington, as for 91/1639-41, May 1977-Apr 1978. 1 ㅇ, J, 91/1647 (WAM), Durokoppin Nature Reserve, $31^{\circ} 30^{\prime} \mathrm{S}$ $117^{\circ} 44^{\prime} \mathrm{E}, 1$ Mar 1988, D. Mitchell et al., beating. 1 ơ $^{\dagger} 1$ ㅇ, 91/1644-46 (WAM), as for 91/1647, 10 Dec 1987. 1 \% , 91/1648 (WAM), Ferndale (suburb of Perth), $33^{\circ} 49^{\prime}$ S $115^{\circ} 57^{\prime} E, 18$ Jan 1978, D. Connop; among grape vine leaves. 1 9 , 91/1649 (WAM), $3.2 \mathrm{~km}$ E of Gin Gin, 26 Nov 1968, Hale School. 19 , 91/1654 (WAM), Lyons R., $24^{\circ} 28^{\prime}$ S $117^{\circ} 14^{\prime} \mathrm{E}$, 29 Jul 1989, AEJ. 1 9, 91/1693 (WAM), Parmelia, $32^{\circ} 15^{\prime}$ 'S $115^{\circ} 47^{\prime} \mathrm{E}, 26$ Jan 1991, AEJ. 1 \% , 91/1692 (WAM), 30 Dec 1989, as for 91/1693; on lemon tree. $19,91 / 1694$ (WAM), Tim Ealey Hill, $21^{\circ} 35^{\prime} \mathrm{S} 118^{\circ} 59^{\prime} \mathrm{E}, 21$ Apr 1989, M.S. Harvey. 2 9 , 92/108-9 (WAM), Bold Park, 31 56 'S $115^{\circ} 46^{\prime} \mathrm{E}, 4 \mathrm{Feb} 1987$, W.F. Humphreys. $10^{\star}, 1$ ㅇ, 92/111-2 (WAM), as for 92/108-9, 10 Mar 1987. 1 ㅇ, N1992173-4 (SAM), Watheroo, $30^{\circ} 18^{\prime} \mathrm{S}$ $116^{\circ} 03^{\prime} \mathrm{E}, 29$ Sep 1981, DH. TASMANIA: $3 \widehat{o}^{\widehat{~}}$, Hickman Collection (AMS), Hobart, $42^{\circ} 50^{\prime} \mathrm{S} 147^{\circ} 15^{\prime} \mathrm{E}, \mathrm{VVH}$. NORFOLK ISLAND: $19,3 j$, N1981239 (SAM), 29 $05^{\prime} \mathrm{S} 168^{\circ} 00^{\prime} \mathrm{E}$, December 1915 to January 1916, A.M. Lea.

Diagnosis. CL female 2.8-4.7, male 2.4-3.5. Cribellum divided. Separated from $P$. vandiemeni by distal spermathecal lobe usually rounded or transversely ovoid and interlobar duct not recurved distally. Male palp often without an obvious patellar apophysis. Late instar juveniles and adults found in communal and solitary webs. 

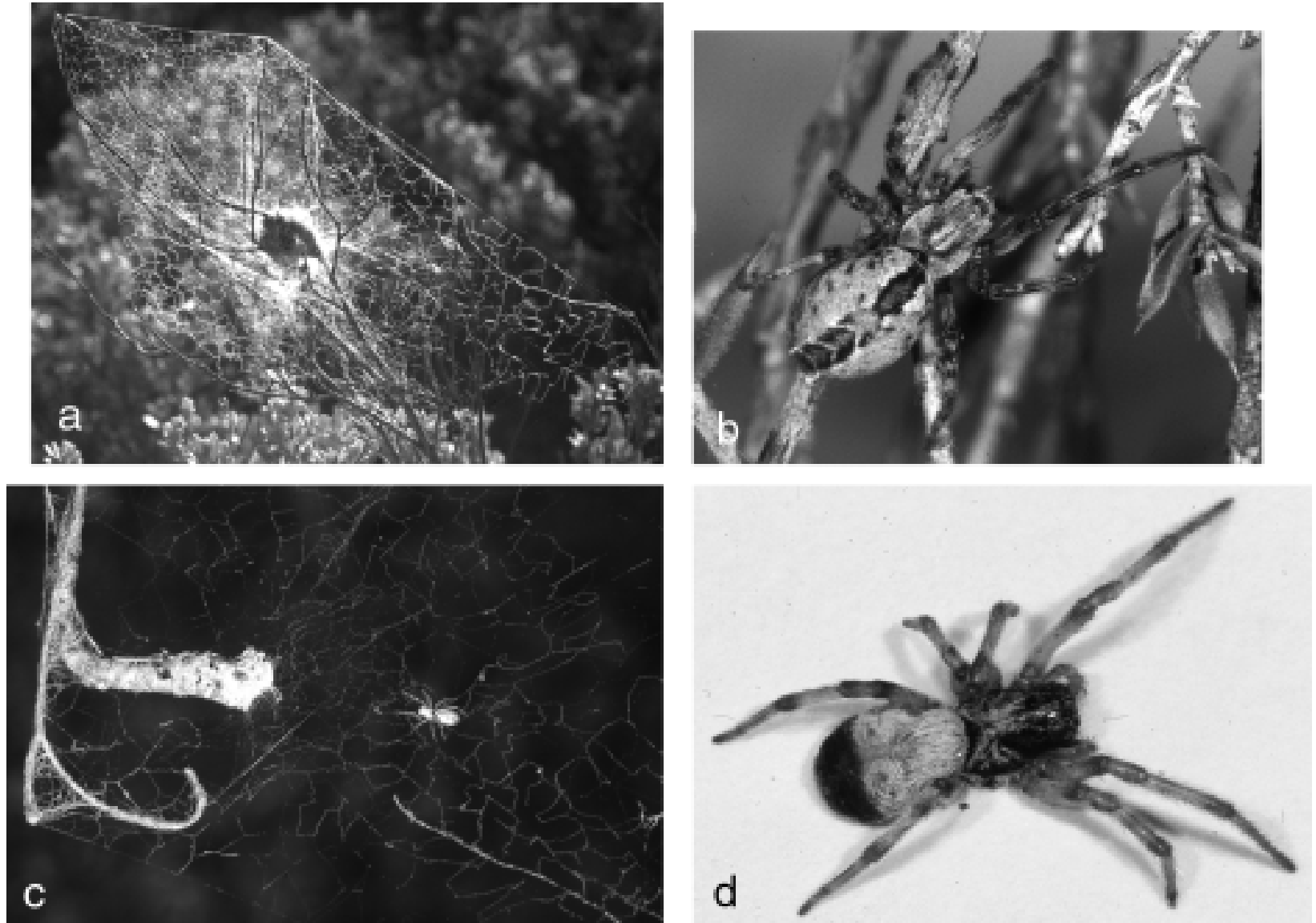

Figure 3. a-d, spiders and webs. a,b, Phryganoporus candidus: a, communal web; b, female. c, Phryganoporus nigrinus: female on solitary web with horn-shaped retreat. d, Phryganoporus melanopygus: female (note partial loss of white setae on carapace).

\section{Description}

Male (KS6937). BL 6.25, CL 2.88, CW 2.10, LL 0.46, LW 0.64 , SL 1.49, SW 1.32. Shape and colouration similar to female. Spination: leg I, femur d112 0011, tibia v022 p101 r101, metatarsus d002 v221 p011 r0101; leg II, femur d112 p011, tibia v012 p011 r0101, metatarsus d012 v121 p011 r0101; leg III, femur d113, tibia v002 p11 r11, metatarsus d12 v121 p11 r11; leg IV, d112, tibia v012 p101 r101, metatarsus d002 v121 p011 r001. Male palp with central limb of " $\mathrm{S}$ " shaped embolus/conductor slanting diagonally across bulb; apical section of "S" narrow, about half as wide as basal section. Palpal patella usually without an apophysis, but a small conical to digitiform apophysis may be present; white, spatulate hairs present or absent.

Female (KS6936). BL 7.80, CL 3.68, CW 2.54, LL 0.37, LW 0.46, SL 1.81, SW 1.46. Carapace colour silvery grey to greyish-brown. Abdomen light grey-brown with brown patterning. Anterior mid-dorsal stripe dark grey-brown, lightened by white hairs centrally, and bordered by white hair patches. Behind the stripe is a row of 4-5 chevrons, as wide or wider than the stripe, each coalescing centrally, the first chevron poorly defined, usually much lighter brown than the rest. Remaining chevrons are brown centrally and variably bordered with darker grey-brown pigment, each limited anteriorly by a line of white hairs that terminate laterally as white tufts. Spination: leg I, femur d1212 p111, tibia v222 p101 r0101, metatarsus d0102 v221 p011 r101; leg II, femur d1202 p111, tibia v122 p101 r101, metatarsus d102 v221 p0101 r101; leg III, femur d133, tibia v122 p11 r11, metatarsus d112 v221 p101 r101; leg IV, femur d113, tibia v112 p11 r11, metatarsus d122 v221 p011 r001. Transverse epigynal ridge broad, often indented anteriorly, sometimes posteriorly also, longitudinal width one-third to one-fifth as wide as epigynal fossa. Fossa as long as wide or anteriorly wider than long. Lateral teeth usually broadly pointed. Female genitalia with short, interlobar ducts curving laterally between the central and distal spermathecal lobes. Distal lobe usually rounded to transversely ovoid; longitudinally ovoid in some populations (Fig. 5f,i).

Distribution. Mainland Australia (Fig. 4a) and Norfolk Island in open forest to shrubland habitats. A single collection from Hobart, Tasmania (Hickman Collection, AMS) may be anthropogenic.

Variation. Genitalic and palpal variation is particularly evident in southern mainland populations, potentially making difficult the assignment of individuals between the very similar species, $P$. candidus and P. vandiemeni. Variation is notable in the orientation of the distal spermathecal lobes (Fig. 5f,i) and in the development of a male palpal patellar apophysis - vestigial to absent or present (Fig. 5e,g). The two character states, "longitudinal" distal spermathecal lobes and well-developed patellar apophyses, are typically associated, as in the Wee Jasper and Black Mountain populations of southeastern Australia. These spiders are found in both small communal and solitary web retreats and were regarded as representatives of $P$. gausapatus by Gray (1983). However, genetically they are identified with $P$. candidus and form a group within the " $B$. candida clade" of Colgan \& Gray (1992). 

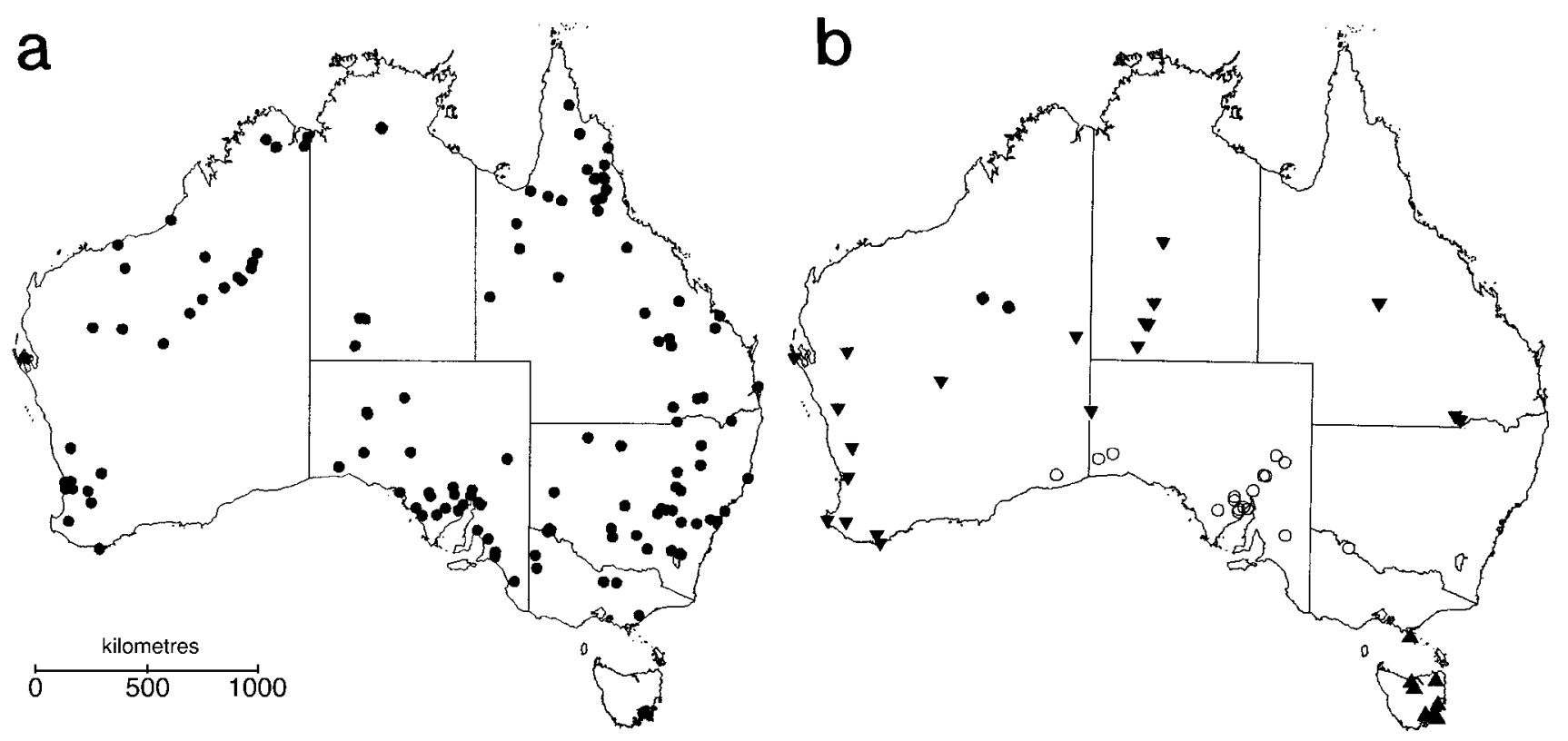

Figure 4. a,b, species distributions based on material examined: a, $\bullet$ Phryganoporus candidus; b, $\mathbf{\nabla}$ P. nigrinus, $\circ$ P. davidleei, $\bullet P$. melanopygus, $\triangle P$. vandiemeni.

Some males from populations in the St George and Drummond Range areas of S.E. Queensland are unusual in lacking most or all abdominal chevron patterning (which is largely retained in females), and in possessing slightly narrowed palpal cymbia. Examination of more material is needed to confirm whether they represent a new candidus group species.

Biology. A detailed study of the biology and behaviour of P. candidus is given by Downes (1993, 1994a,b) (see introductory notes). Communal nests observed by the present author varied from a few centimetres to about 30 $\mathrm{cm}$ across (Fig. 3a). Most were built on sclerophyllous, often spiky, shrubs and small trees such as mallee eucalypts. The largest $P$. candidus communal nest observed contained 558j, subadults and adults-40 $\hat{0}$ and $23 \%$ (KS17177). Small, solitary sheet webs are also common, the retreat consisting of a thick-walled silk tube (Gray, 1983, fig. 36), usually twisted around the plant stem with the mouth opening downward or to the side. The small, lenticular egg sacs contain from 13-39 eggs.

\section{Phryganoporus vandiemeni (Gray) n.comb.}

Figs. $4 \mathrm{~b}, 5 \mathrm{j}-1$

Badumna vandiemeni Gray, 1983. Holotype male, Eaglehawk Neck, Tasmania, $43^{\circ} 01^{\prime}$ S $147^{\circ} 55^{\prime}$ E, 3 Jul 1980, MRG (KS6976, AMS-examined).

Ixeuticus candidus Hickman, 1967: 33, figs. 50, 51.

Badumna vandiemeni Gray, 1983: 257, figs. 29-34; Colgan \& Gray, 1992: 86; Platnick, 1989: 408.

Material examined. TASMANIA: female paratypes: KS6977, data as for holotype; KS6978, data as for holotype, 6 Mar 1960, VVH; J763 (TM), Lauderdale, $42^{\circ} 54^{\prime} \mathrm{S} 147^{\circ} 30^{\prime} \mathrm{E}$, April 1971. 1 우 KS6042, 8 Mar 1960, other data as paratype KS6978. $8 j$, KS6034, February 1948, in webs on shrubs, other data as above. $1 \hat{\delta}, 1 j$, KS19144, Eaglehawk Neck, Taranna area, Tas., $43^{\circ} 02^{\prime} \mathrm{S} 147^{\circ} 53^{\prime} \mathrm{E}, 27$ Mar 1988, MRG; in solitary nests on Acacia uricifolia. 1 1 , KS19146, West of Copping, Tas., 42 ${ }^{\circ} 49^{\prime} \mathrm{S}$ $147^{\circ} 45^{\prime} \mathrm{E}, 27$ Mar 1988, MRG; on gorse bushes. 1 q , 4j, KS23452, outside "Liberty Castle", $18 \mathrm{~km} \mathrm{~S}$ of Devonport, Tas., $41^{\circ} 17^{\prime} \mathrm{S} 146^{\circ} 30^{\prime} \mathrm{E}, 4 \mathrm{Nov}$
1990, JT; in remnant vegetation (solitary nest on Acacia sp). 10 3 , 9 , KS29076, Lenah Valley, Tas., $42^{\circ} 52^{\prime} \mathrm{S} 147^{\circ} 17^{\prime} \mathrm{E}, 22$ May 1923, VVH; in webs on shrub. $4{ }^{\star}, 1 j$, KS29094, Cascades, Tas., $41^{\circ} 10^{\prime} \mathrm{S} 147^{\circ} 49^{\prime} \mathrm{E}, 6$ Apr 1953, VVH. $1 \stackrel{9}{\circ}, 1 j$, KS31606, $14 \mathrm{~km} \mathrm{~N}$ of Triabunna, Tas., $42^{\circ} 21^{\prime} \mathrm{S}$ $147^{\circ} 56^{\prime} \mathrm{E}$, October 1990, MRG; on Acacia (prickly moses), solitary web. 19 , KS34455, Bruny Island, Alonnah area, Tas., $43^{\circ} 16^{\prime} \mathrm{S} 147^{\circ} 19^{\prime} \mathrm{E}, 28$ Oct 1990, MRG, CH \& JT; in web on prickly pea. $19,2 j$, KS34470,

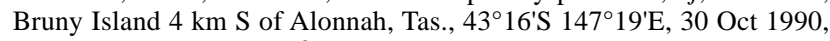
MRG; in solitary nests. 3 \% , KS45065, Electrona, Tas., $43^{\circ} 02^{\prime} \mathrm{S} 147^{\circ} 16^{\prime} \mathrm{E}$, October 1990, MRG, CH, JT. VICTORIA. 10, 4 ㅇ, KS31663, Wilson's Promontory, $39^{\circ} 05^{\prime} \mathrm{S} 146^{\circ} 25^{\prime} \mathrm{E}$, April $1990, \mathrm{MRG}$.

Diagnosis. CL female 2.9-4.0, male 4.0-4.4. In solitary webs. Cribellum divided. Distinguished from P. candidus females by the distally recurved interlobar spermathecal ducts; and by the combined presence of longitudinally ovoid distal spermathecal lobes, a male palpal patellar apophysis and solitary webs. Found in Tasmania and far southeast mainland Australia.

\section{Redescription}

Male (KS6976, holotype). BL 8.55, CL 4.06, CW 2.80, LL 0.27 , LW 0.34, SL 0.96, SW 0.78. Similar to female. Male palpal bulb and tibia like $P$. candidus. Palpal patella with a conical, retrodorsal apophysis, adorned with several white, spatulate hairs (Fig. 5 1).

Female (KS6977, paratype). BL 8.60, CL 3.69, CW 2.48, LL 0.54, LW 0.60, SL 0.88, SW 0.75. Similar to P. candidus. Posterodorsal abdominal patterning sometimes lighter, chevrons comprising light brown patches with limited dark brown edging. Lateral tufts of white hairs on chevrons well developed. Epigynal structure as for $P$. candidus. Internal genitalia with longitudinally ovoid distal spermathecal lobes and distally recurved interlobar ducts (Fig. 5k).

Distribution. Tasmania and far southern Victoria in open forest and heathland (Fig. 4b).

Biology. These spiders are known only from solitary webs These webs are commonly associated with sclerophyllous, 

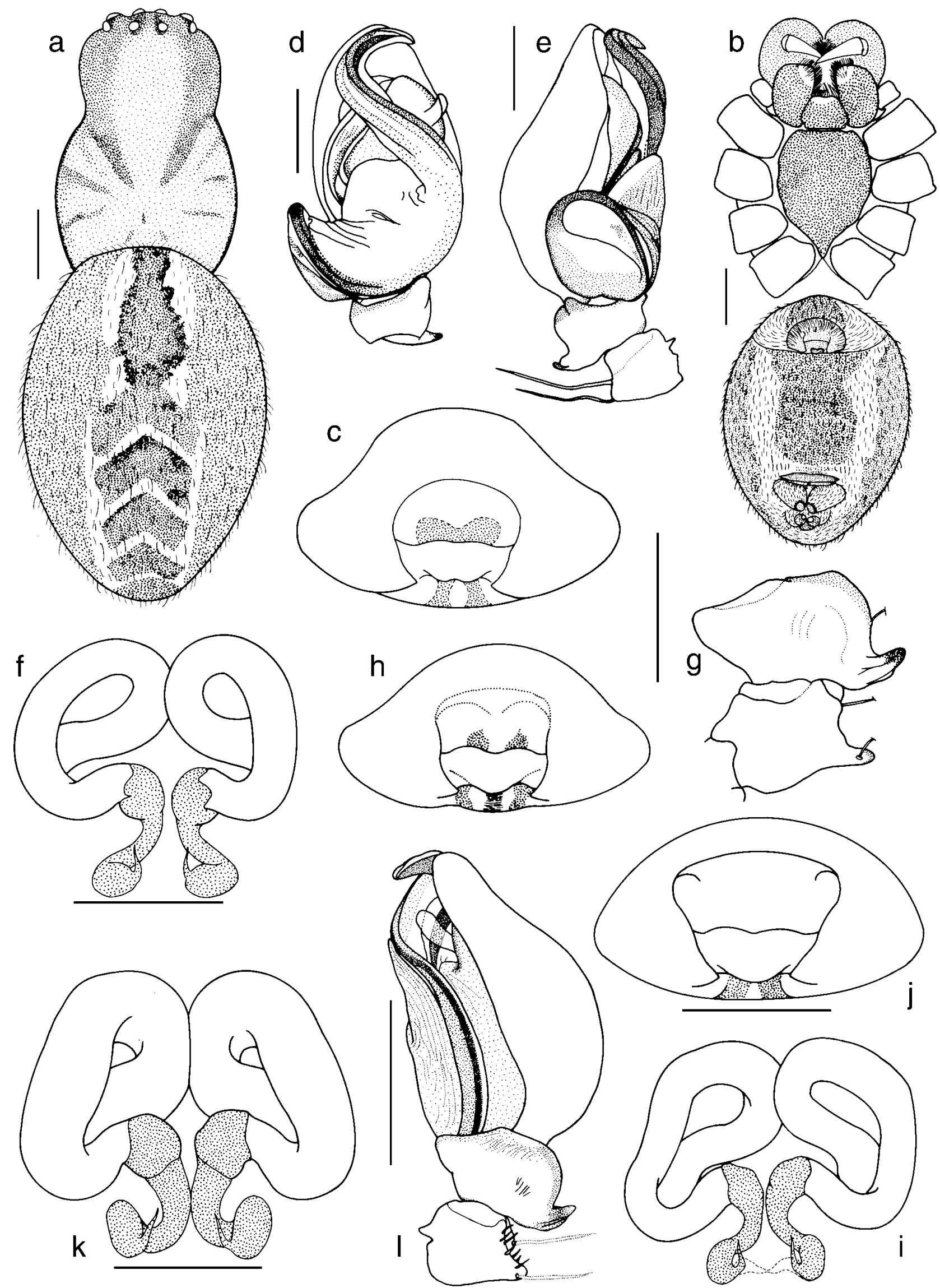

Figure 5. a-i, Phryganoporus candidus. a-c, Townsville, Qld, female: a, body, dorsal; b, body, ventral; c, epigynum. d,e, Grenfell, NSW, male palp: d, ventral; e, prolateral. f, Mellin Jerrie Well, WA, female: internal genitalia. g-i, Black Mountain, ACT: g, male palp, retrolateral tibia \& patella; h,i, female: h, epigynum; i, internal genitalia. j-1, Phryganoporus vandiemeni, Wilson's Promontory, Vic.: j,k, female: j, epigynum; k, internal genitalia. 1, male palp, retrolateral. Scale lines: a, b: $1 \mathrm{~mm}$; others $0.5 \mathrm{~mm}$. 
often spiky, shrubs, including Acacia spp. The web typically consists of a small, irregular cribellate sheet radiating from a central retreat, consisting of a "cowl" of silk forming a roof over a wide entrance funnel opening to the side or down, and leading back into a thick-walled silk retreat tube twisted around a stem. The specimens examined suggest that male spiders are as large or larger than females, in contrast to other species.

Remarks. Electrophoretic studies show that $P$. vandiemeni has fixed differences at three loci and is highly differentiated at a fourth locus (Colgan \& Gray, 1992). These studies have also confirmed its presence on the mainland at Wilson's Promontory, Victoria, a southern coastal area formerly connected via a land bridge to Tasmania. The southern mainland distribution of this species may prove to be more extensive, but determination of this will probably require further molecular studies.

\section{Phryganoporus davidleei n.sp.}

Figs. 4b, 6a-h

Type material. HolotyPE male, N1991365 (SAM), Pinkawillinie Conservation Park, Eyre Peninsula, South Australia, $33^{\circ} 07^{\prime} \mathrm{S} 136^{\circ} 00^{\prime} \mathrm{E}, 19-20$ May 1990, D. Hirst. PARATYPES South Australia: 20 , 1 9 , N1991361-3 (SAM), data as for holotype, 21 May 1990. $1 \hat{o}^{\hat{0}}$, KS45778, data as for holotype, 8-19 May 1990. 1 q , N1991378 (SAM), Bunyeroo Gorge, Heysen Range, $31^{\circ} 25^{\prime} \mathrm{S} 138^{\circ} 32^{\prime} \mathrm{E}, 16$ May 1990, DH

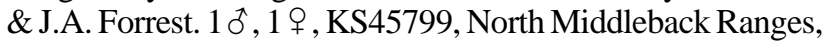
$33^{\circ} 04^{\prime} \mathrm{S} 137^{\circ} 08^{\prime} \mathrm{E}$, June 1984 , BG; on Daviesia in mallee woodland. Western Australia: 19 , KS17138, Nullarbor Plain,

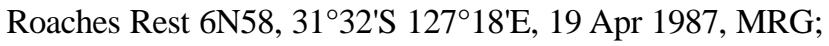
solitary retreat with egg sac on doline shrub.

Other material examined. South Australia: $2 \hat{0}$, N1991370-1 (SAM), Lake Dam near Lake Frome, 3044'S $139^{\circ} 34^{\prime} \mathrm{E}, 10$ May 1989, DCL. 19, N1992143 (SAM), S of Middleback Station, $32^{\circ} 57^{\prime} \mathrm{S} 137^{\circ} 23^{\prime} \mathrm{E}$, June 1984 , BG. 1 우, $1 j$, N1991360 (SAM), $20 \mathrm{~km} \mathrm{~N}$ of Cook, 30 $27^{\circ} \mathrm{S} 130^{\circ} 19^{\prime} \mathrm{E}$, 18 Aug 1980, G.J. Baker. 19, j, N1991366 (SAM), Lake Gilles Conservation Park, $33^{\circ} 05^{\prime} \mathrm{S} 136^{\circ} 38^{\prime} \mathrm{E}, 24-25$ Sep 1988 , DH; nest \& spiderlings. $19,1 j$, N1991367 (SAM), locality as above, 2 Apr 1987, DCL, DH; in solitary silk nests on dead shrubs. 19 , N1991368 (SAM), Whyalla Sewerage pond, 330.'S 137³3'E, 27 Jul 1975, P Hudson. 1 + , N1991369 (SAM), $40 \mathrm{~km} \mathrm{~N}$ of Port Augusta, 32 $08^{\circ} \mathrm{S} 137^{\circ} 52^{\prime} \mathrm{E}, 27$ May 1986, H. Mincham. 20, 3 ㅇ, N1991372-6 (SAM), Gammon Ranges towards Elephant Hill from Arcoona Ck, 30 $25^{\circ} \mathrm{S}$ $139^{\circ} 05^{\prime} \mathrm{E}, 5$ May 1989, DH. 19 , N1991377 (SAM), Bunyeroo Ck, ABC Range, $31^{\circ} 25^{\prime} \mathrm{S} 138^{\circ} 34^{\prime} \mathrm{E}, 14-15$ May 1990 , DH. 10 , 1 ㅇ, N1992137-8 (SAM), S of Middleback Station, $32^{\circ} 57^{\prime} \mathrm{S} 137^{\circ} 23^{\prime} \mathrm{E}$, June 1984, BG; in web case on Muelenbechia cunninghamii. 10, 3 \% , N1992139-42 (SAM); from solitary nests. $1+$, N1992144 (SAM), data as for N1992137/ 8. 1 \%, N1992145 (SAM), South Middleback Ranges, $33^{\circ} 11^{\prime} \mathrm{S}$ $137^{\circ} 06^{\prime} \mathrm{E}$, 4 Jun 1984 , BG. $19,1 j$, N1992148-50 (SAM), Hughes, $30^{\circ} 43^{\prime} \mathrm{S} 129^{\circ} 31^{\prime} \mathrm{E}$, Sep 1984, BG; with spiderlings, web on Atriplex. 1 ㅇ, N1992156 (SAM), Roonka, 341'' S $139^{\circ} 38^{\prime}$ E, $12-14$ Oct 1991, Strathalbyn Field Naturalists. VICTORIA: $2{ }^{\star}, 2$, , N1992178-81 (SAM), south of Red Cliffs, $34^{\circ} 20^{\prime}$ S $142^{\circ} 14^{\prime} \mathrm{E}$, May 1979, DH.
Diagnosis. Cribellum entire. CL female 1.9-3.5, male 1.83.1. Separated from P. nigrinus and melanopygus by the presence of free abdominal chevrons (pigmentation variable, moderate to weak).

\section{Description}

Male holotype. BL 4.28, CL 2.01, CW 1.38, LL 0.26, LW 0.37 , SL 1.04, SW 0.83. Similar to female. Spination: leg I, femur d111, p01, tibia v012 p011, metatarsus v221 p11 r11; leg II, femur d111 p01, tibia v12 p11, metatarsus v221 p11 r11; leg III, femur d10 p01, tibia v02 p01 r01, metatarsus d02 v111 p11 r11; leg IV, femur d10, tibia v02 r01, metatarsus d02 v01 p111 r01. Central limb of "S" shaped embolus/conductor passing horizontally across palpal bulb; apical section of " $\mathrm{S}$ " relatively broad, more than threequarters width of basal section. Prolateral tegular bulge height often more than half cymbium length.

Female paratype (KS17138). BL 7.11, CL 3.19, CW 2.13, LL 0.49, LW 0.60, SL 1.54, SW 1.21. Carapace with numerous white hairs, overall colour varying from silvery grey to light brown. Abdomen light grey to brown with dense, recumbent white hair cover, dark brown erect hairs mostly confined to brown pigment flecks. Dorsal abdomen with a dark brown anterior stripe but posterior chevrons mostly lighter brown, occasionally poorly defined. Chevrons not fused. Legs light brown with dark brown pigment rings. Spination: leg I, femur d0110 p0011, tibia v010, metatarsus v222 p010 r0101; leg II, femur d0110 p0001 r0001, tibia v010 p0010, metatarsus v111 p011 r0101; leg III, femur d100 r001, tibia v001 r01, metatarsus v021 p012 r012; leg IV, femur d100, tibia v011 r01, metatarsus v102 p001 r002. Transverse epigynal ridge moderately procurved and narrow, longitudinal width less than one-fifth as wide as epigynal fossa. Fossa wider than long. Lateral teeth narrowly pointed. Internal genitalia Fig. 6g.

Distribution. Central southern Australia in open forest to shrubland (Fig. 4b).

Biology. Documented webs are solitary, consisting of small sheet webs built on shrubs, retreat structure poorly documented, but probably similar to $P$. candidus solitary webs.

Etymology. The specific epithet honours David Lee, former research acarologist and Curator of Arachnology \& Helminths at the South Australian Museum.

\section{Phryganoporus nigrinus Simon}

Figs. 1b-f,h-j, 2g-j, 3c, 4b, 7a-h, 8a-i

Phryganoporus nigrinus Simon, 1908: 368. Holotype female (MNHN) from Boyanup, Western Australia, 1905 (Stat. 146, Michaelsen and Hartmeyer Expedition to Southwest Australia). Ixeuticus candidus.-Main, 1971: 119.

Badumna candida.-Gray, 1983: 250, figs. 9, 37.

Material examined. NORTHERN TERRITORY: 10 , KS55813, Watarrka National Park, King's Canyon, $24^{\circ} 15^{\prime} \mathrm{S} 131^{\circ} 31^{\prime} \mathrm{E}, 16$ May 1999, MRG, GAM \& HMS; solitary web with horn-shaped retreat on dead shrub. 19 , KS55814, 1 \%, KS55833, data as KS55813. 1 \% , KS55818, Watarrka National Park, Kathleen Springs, 2420'38"S 131 ${ }^{\circ} 40^{\prime} 54 " E, 15$ May 1999, MRG, GAM \& HMS; in horn-shaped retreat on dead shrub. 1 \% , KS55819, 19 , KS55820, data as KS55818. 1 đ, KS55812, data as KS55818 except

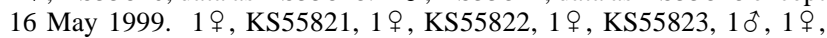



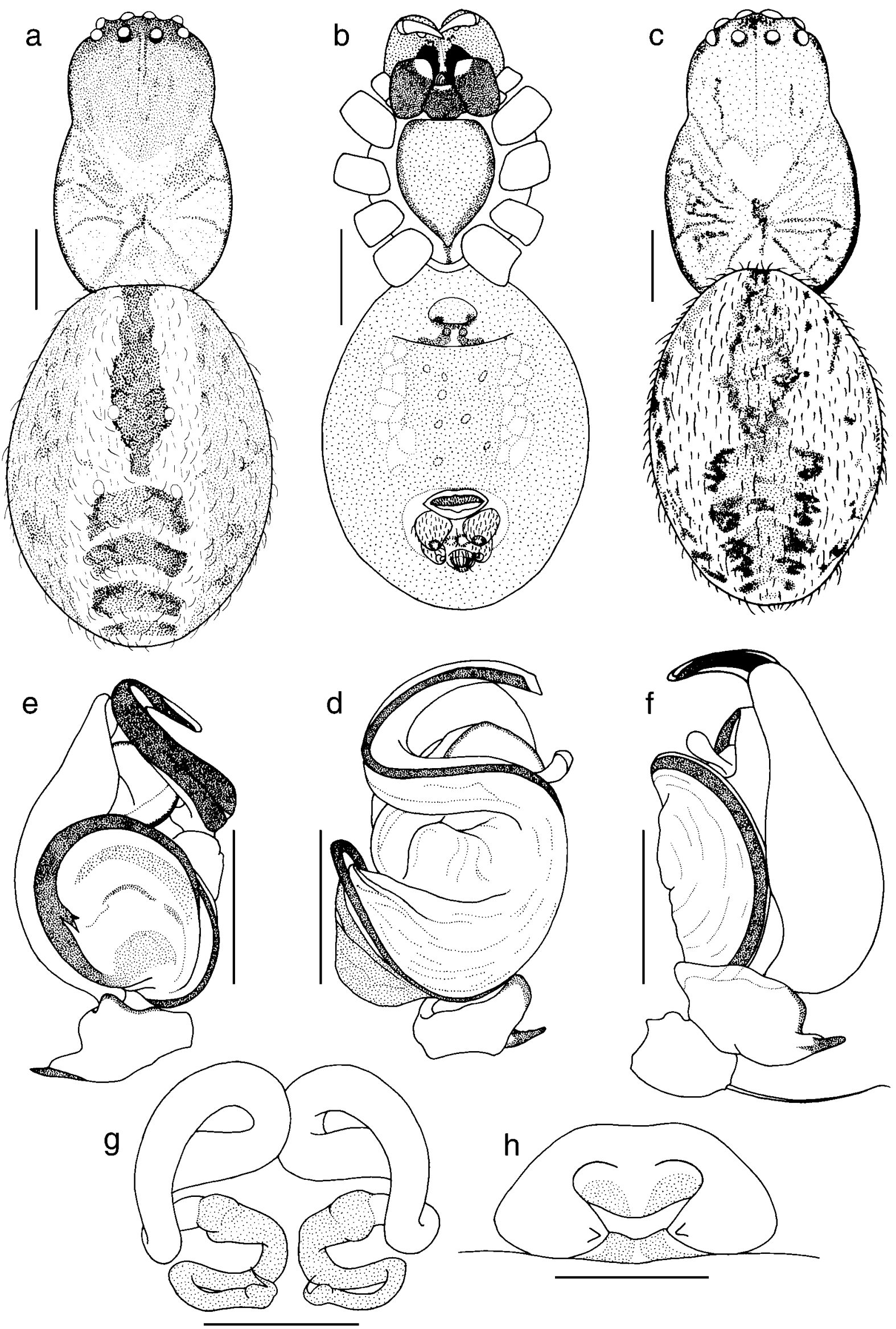

Figure 6. a-h, Phryganoporus davidleei n.sp. a,b,g,h, females. a,g, Roaches Rest, WA: a, body, dorsal, g, internal genitalia. b,h, Bunyeroo Gorge, SA: b, body, ventral; h, epigynum. c-f, Pinkawillinie, SA, male: c, body, dorsal; d-f, palp, d, ventral, e, prolateral, f, retrolateral. Scale lines: a-c: $1 \mathrm{~mm}$; others $0.5 \mathrm{~mm}$. 

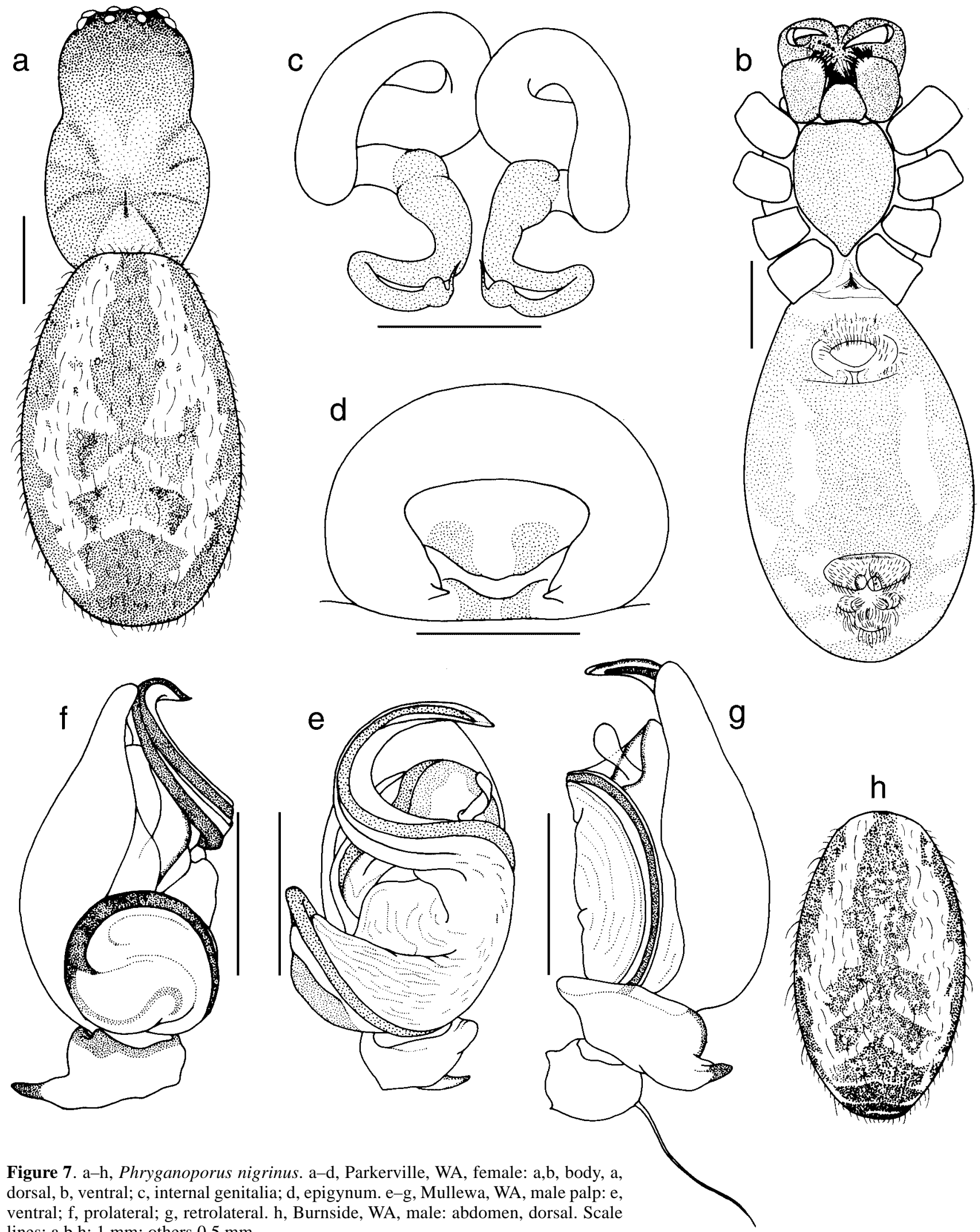

Figure 7. a-h, Phryganoporus nigrinus. a-d, Parkerville, WA, female: a,b, body, a, dorsal, b, ventral; c, internal genitalia; d, epigynum. e-g, Mullewa, WA, male palp: e, ventral; f, prolateral; g, retrolateral. h, Burnside, WA, male: abdomen, dorsal. Scale lines: a,b,h: $1 \mathrm{~mm}$; others $0.5 \mathrm{~mm}$.

KS55824, data as KS55812. 1 우 KS55815, $1 \mathrm{~km}$ E of Glen Helen, Namatjira Drive, West MacDonnell Ranges National Park, 2340'57"S $132^{\circ} 40^{\prime} 50 "$ "E, 17 May 1999, MRG, GAM \& HMS; horn-shaped retreat. 1 ․ KS55816, Uluru National Park, 6 km SSW of Ranger Station,
2524'03"S 130 59'18"E, 13 May 1999, MRG, GAM \& HMS; on dead mallee. 1 s 1 우, KS55826, Uluru National Park, Ranger accommodation area, 2521'16"S $131^{\circ} 04^{\prime} 11^{\prime \prime E}, 14$ May 1999, MRG, GAM \& HMS; on prickly acacia. 10 , KS55834, S of Barrow Ck near communications 

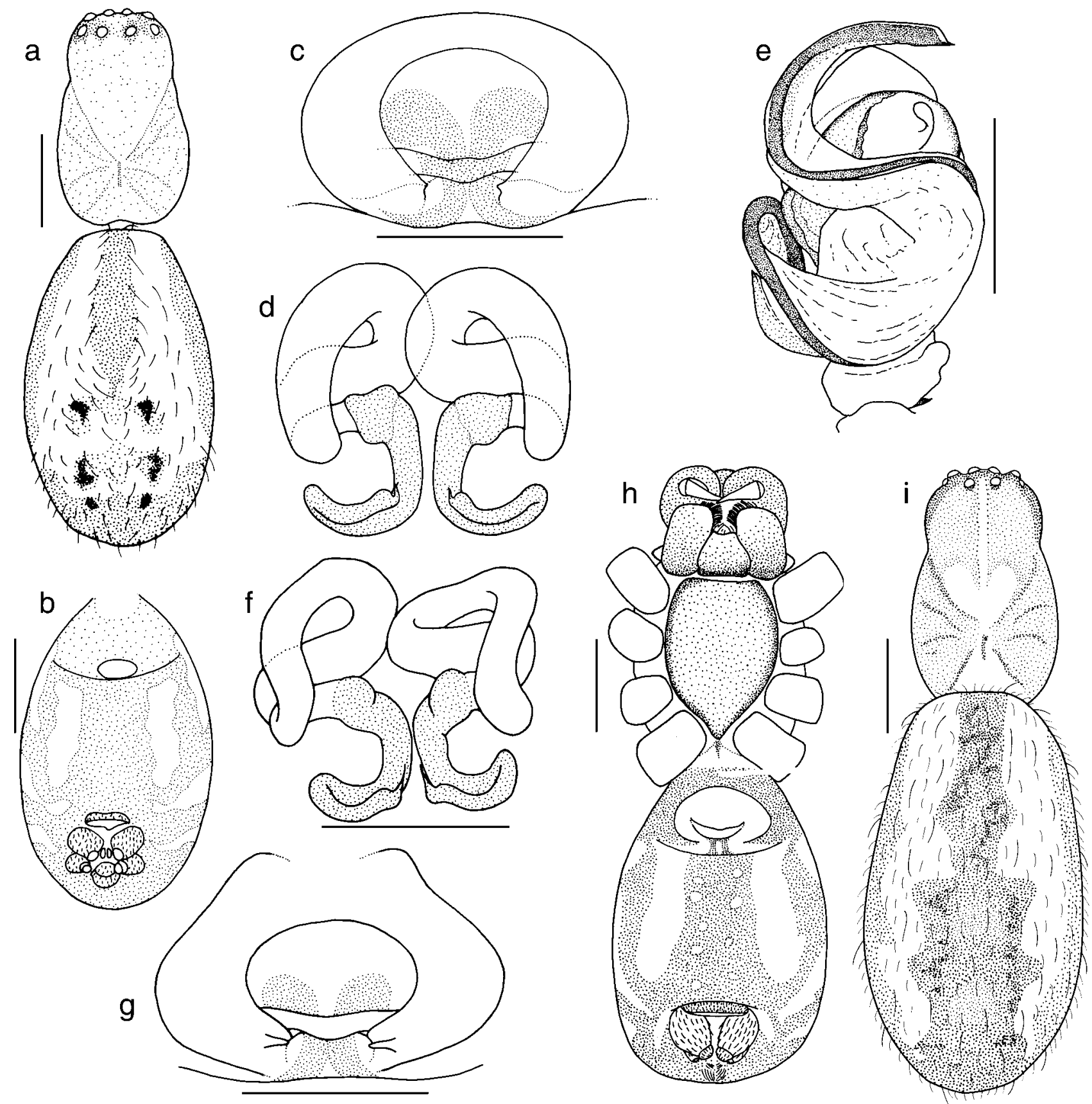

Figure 8. a-i, Phryganoporus nigrinus. a-d, Mungindi, Qld, female: a, body, dorsal; b, abdomen, ventral; c, epigynum; d, internal genitalia. e, Kathleen Springs, N.T.: male palp, ventral. f-i, Great Victoria Desert, SA, female: f, internal genitalia; g, epigynum; h, body, ventral; i, body, dorsal. Scale lines: a,b,h,i: $1 \mathrm{~mm}$; others $0.5 \mathrm{~mm}$.

tower beside Stuart Highway, 2137'43"S 13345'13"E, 18 May 1999, MRG, GAM \& HMS; in short, horn shaped retreat on dead shrub. WESTERN AUSTRALIA: $1{ }^{\star}$, KS6941, Burnside, $2.3 \mathrm{~km} \mathrm{~N}$ of Witchcliffe $\mathrm{Rd}$ turn off on Caves Rd, Margaret R. region, $33^{\circ} 56^{\prime} \mathrm{S} 115^{\circ} 01^{\prime} \mathrm{E}, 26 \mathrm{Jan}$ 1979, MRG; retreat a horn-shaped tube ("dark colour variant of $B$. candida" in Gray, 1983). 1 ते, KS6984, Wooderarrung Ck, $3 \mathrm{~km} \mathrm{~N}$ of Mullewa, 28 32'S 115 30'E, 22 Feb 1979, MRG; on Acacia brachystachya. 1 , 91/1691 (WAM), Parkerville, 31 ${ }^{\circ} 53^{\prime} \mathrm{S} 116^{\circ} 08^{\prime} \mathrm{E}$, October 1976, R. Browne; horn shaped retreat. 10 , 2 \%, 2j, N1992175-7 (SAM), Albany, $35^{\circ} 00^{\prime} \mathrm{S} 117^{\circ} 52^{\prime} \mathrm{E}, 27-30$ Oct 1981, DH (in separate webs). 1ㅇ, BY19 (AMS), Albany Highway on Capella Rd, 28 Jul 1984, BYM. 1 \%, 91/1638 (WAM),

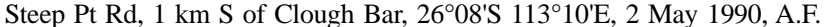
Longbottom. 1, 91/1656 (WAM), $14 \mathrm{~km} \mathrm{SW}$ of Bridgetown on Mockerdillup Rd, $34^{\circ} 01^{\prime}$ S $116^{\circ} 00^{\prime} E, 27$ Aug 1987, J.M. Waldock. 1ㅇ, 32j, 91/1657-90 (WAM), Mt Barker, S of Lookout, 34 $38^{\circ}$ 'S $117^{\circ} 40^{\prime} \mathrm{E}, 18$ Nov 1990, A.F. Longbottom. 19, 91/1695 (WAM), Wal-Arrie Pool, 254'S $115^{\circ} 58^{\prime} \mathrm{E}, 7$ May 1989, D. Knowles. $19,91 / 1610$ (WAM), Barwidgee Station, $2 \mathrm{~km}$ E of Little Well, $27^{\circ} 08^{\prime} \mathrm{S} 121^{\circ} 00^{\prime} \mathrm{E}, 16$ May 1990, S. Gilligan. $29,2 j$, 91/1650-3 (WAM), Glen Cummings Gorge, $25^{\circ} 02^{\prime} \mathrm{S} 128^{\circ} 18^{\prime} \mathrm{E}, 13-14$ Jan 1990, M.S. Harvey \& T.F. Houston; in solitary webs. SoutH AuSTRALIA: 1 , N1991356 (SAM), Great Victoria Desert, August 1980. 1 \& ,j, N1991357 (SAM), Great Victoria Desert, $175 \mathrm{~km}$ W of Vokes Hill Corner, 28 $30^{\circ} \mathrm{S}$ $12^{\circ} 01^{\prime}$ E, 25 Aug 1980, G.J. Baker. 1 9 , N1991358-9 (SAM), data as for N1991357; conical, webbed nest on Acacia colletoides. QUEENSLAND: 1 \%, KS42616, $12 \mathrm{~km} \mathrm{~N}$ of Mungindi beside Carnarvon Highway, 28 $53^{\circ} \mathrm{S}$ $148^{\circ} 57^{\prime} \mathrm{E}, 25$ Apr 1994, M. Moulds \& JT; eucalypt woodland. 29 , KS45062 \& KS45119, roadside rest area $1 \mathrm{~km} \mathrm{E}$ of Barcaldine, $23^{\circ} 33^{\prime} \mathrm{S} 145^{\circ} 18^{\prime} \mathrm{E}, 9$ Jul 1995, JT, M. Tio, S. Cowan; solitary webs, on dead spiky bush (KS45062) and bottlebrush (KS45119). $59, \mathrm{KS} 44167$, roadside rest area $1 \mathrm{~km} \mathrm{E}$ of Barcaldine, $23^{\circ} 33^{\prime} \mathrm{S} 145^{\circ} 18^{\prime} \mathrm{E}, 1$ Aug 1995 , JT; solitary webs on dead bushes. 1 , KS44158, $30 \mathrm{~km} \mathrm{~S}$ of St George, 28 $8^{\circ} 12^{\prime} \mathrm{S} 148^{\circ} 44^{\prime} \mathrm{E}, 2$ Aug 1995, JT. 
Diagnosis. CL female 2.2-3.1, male 1.8-2.5. Cribellum entire. Separated from $P$. davidleei by the dark brown posterodorsal abdominal patch and from $P$. melanopygus by the rather cylindrical body shape and the horizontal central limb of the S-shaped embolus/conductor. In solitary webs with tapering, horn-shaped retreats (Fig. 3c).

\section{Description}

Male (KS6941). BL 5.55, CL 2.44, CW 1.65, LL 0.37, LW 0.42 , SL 1.22, SW 0.98. Similar to female. Spination: leg I, femur d112 p0011, tibia v122 p11 r11, metatarsus d002 v221 p011 r011; leg II, femur d112 p001, tibia v112 p11 r01, metatarsus d002 v221 p11 r11; leg III, femur d122 p001, tibia v12 p10 r11, metatarsus v221 p01 r11; leg IV, femur d002 v121 r01, tibia d012 r01, metatarsus d002 v221 p011 r001. Palp with central limb of "S" shaped embolus/conductor passing horizontally across bulb; apical section of " $\mathrm{S}$ " relatively broad, about two-thirds width of basal section. Prolateral tegular bulge height less than half cymbium length.

Female (WAM 91/1691). BL 7.23, CL 3.05, CW 1.84, LL 0.46 , LW 0.49, SL 1.49, SW 1.14. Body shape more cylindrical than other species. Carapace colour varies from silvery grey to brown, depending on the density of white hairs present. Abdomen silvery grey-brown laterally with both numerous recumbent white hairs and scattered dark brown flecks, the latter increasing in density ventrolaterally. Anterior mid-dorsal stripe broad, dark brown and coalescent behind with the first chevron marking. The remaining four to five chevrons are dark brown, free anteriorly but partially or fully fused posteriorly, forming a dark patch on the posterodorsal abdomen. Legs with wide, brown-black pigment rings. Spination: leg I, femur d111 p001, tibia v012 p01010, metatarsus v221 p0101 r0101; leg II, femur d110 p001, tibia v011 p01010, metatarsus d002 v221 p0101 r0101; leg III, femur d101, tibia v001 r0010, metatarsus d002 v221 p0101 r0101; leg IV, femur d100, tibia v011 r0010, metatarsus d001 v111 r001. Transverse epigynal ridge procurved and narrow, longitudinal width less than one-eighth as wide as epigynal fossa. Fossa wider than long. Lateral teeth narrowly pointed. Internal genitalia Figs. 7c, 8d,f.

Distribution. Western Australia, central Australia, western Queensland (Fig. 4b). In open forest, woodland and shrubland.

Variation. Female specimens from the Great Victoria Desert (SAM 1991356-9) were a distinctive light, rusty reddish colour (in alcohol) with all chevrons fused (Fig. 8h). Specimens from Queensland have weakly pigmented abdominal patterning (Fig. 8a) compared with central \& western Australian representatives.

Biology. P. nigrinus occupy solitary sheet webs. The simple, asymmetrical, sheet rarely spans more than $25 \mathrm{~cm}$ and is often found on the dead branches of spindly shrubs. The retreats are of unique shape throughout the large range of this species-elongate, horn-like silk tubes attached along a branch, with the wider entrance usually opening downward, sometimes to the side (Fig. 3c, from the Kimberley region of northwestern Australia; Gray, 1983, fig. 37, from south western Australia [attributed to B. candida]; and Main, 1976, fig. 40a [attributed to Dictyna sp.]).

\section{Phryganoporus melanopygus n.sp.}

Figs. 3d, 4b, 9a-h

Type material. HOLOTYPE $\widehat{\delta}$, KS45120 (AMS), track to Windy Corner, Western Australia, 23 ${ }^{\circ} 30^{\prime} \mathrm{S} 124^{\circ} 35^{\prime} \mathrm{E}, 16 \mathrm{Aug}$ 1990, A.E. de Jong. PARATYPES: 29 (with 5j), KS23446 (AMS); 10 , KS45121 (AMS); all data as for holotype. 19 , 91/1619 (WAM), Canning Stock Route, Western Australia, $23^{\circ} 05^{\prime} \mathrm{S} 123^{\circ} 12^{\prime} \mathrm{E}$, $19 \mathrm{Jul}-1$ Nov 1987 , AEJ; tube nest.

Diagnosis. CL female 2.8-3.5, male 1.9-2.2. Separated from all other species by the strong dark brown pigmentation covering the distal third of the abdomen; and from $P$. nigrinus and $P$. davidleei by the gently distally sloping central limb of the S-shaped embolus/conductor.

\section{Description}

Male holotype. BL 3.95, CL 1.93, CW 1.38, LL 0.24, LW 0.37 , SL 1.00, SW 0.82. Similar to female but usually with two distinct abdominal chevrons, remainder fused posteriorly. Spination: leg I, femur d112 p01, tibia v012 p01 r11, metatarsus d01 v221 p11 r11; leg II, femur d112 p01, tibia v012 p11 r10, metatarsus d02 v211 p011 r11; leg III, femur d112 p01, tibia v02 p01 r01, metatarsus d02 v111 p011 r011; leg IV, femur d111 p01, tibia v02 r01, metatarsus d02 v111 p011 r01. Central limb of "S" shaped embolus/ conductor angled gently upward across the upper palpal bulb. Apical section of "S" relatively broad, about two-thirds the width of basal section. Prolateral tegular bulge height usually less than half cymbium length.

Female paratype (KS23446). BL 7.27, CL 3.29, CW 2.24, LL 0.45, LW 0.65, SL 1.75, SW 1.29. Carapace silvery grey to brown depending on density of white hair cover. Anterior two-thirds of abdomen silvery grey with middorsal stripe present but rendered indistinct in life by dense white hair cover. One or two brown chevrons of variable size present, remainder completely fused so that entire distal third of abdomen is dark brown. Legs brown with dark brown pigment rings. Abdomen may broaden posteriorly. Spination: leg I, femur d111 p001, tibia v122 p1010 r0100, metatarsus d002 v221 p001 r0101; leg II, femur d101 p001, tibia v102 p0010, metatarsus d001 v221 p0101 r0101; leg III, femur d100 p001, tibia 002 r010, metatarsus d001 v2111 p001 r001; leg IV, femur d112, tibia v002 r010, metatarsus d001 v011 p01 r001. Transverse epigynal ridge indented anteriorly, longitudinal width about one-fifth as wide as epigynal fossa. Fossa wider than long. Lateral teeth narrowly pointed. Internal genitalia Fig. 9f.

Distribution. Shrubland habitats of the Great Sandy Desert, Western Australia (Fig. 4b).

Etymology. The specific epithet refers to the darkly pigmented posterior abdomen. 

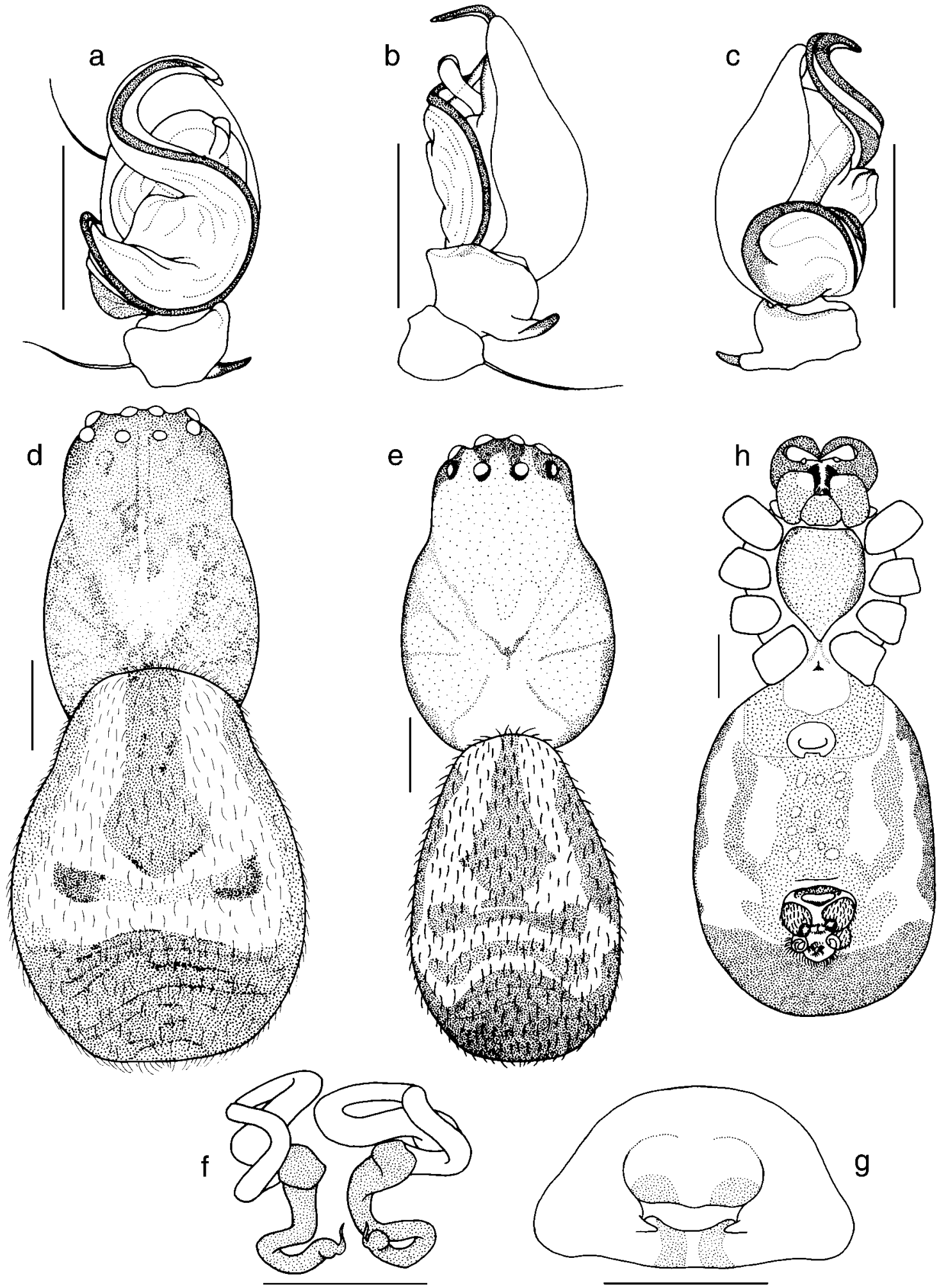

Figure 9. a-h, Phryganoporus melanopygus n.sp. a-g, Windy Corner, WA. a-c, male palp: a, ventral; b, retrolateral; c, prolateral. d,e, body, dorsal: d, female; e, male. f,g, female: f, internal genitalia; g, epigynum. h, Canning Stock Route, WA, female: ventral body. Scale lines: d,e,h: $1 \mathrm{~mm}$; others $0.5 \mathrm{~mm}$. 
ACKNOWLEDGMENTS. Funding assistance from the Australian Biological Resources Study is gratefully acknowledged. Judy Thompson provided artwork assistance. Collections material for this study was kindly provided by David Hirst (SAM), Graham Milledge (NMV), Robert Raven, Phillip Lawless (QM), Mark Harvey, Julianne Waldock (WAM), Barbara York Main (BYM coll.). I am grateful to Mr A.de Jong for provision of specimens from northwestern Australia.

\section{References}

Bond, J.E., \& B.D. Opell, 1997. Systematics of the spider genera Mallos and Mexitlia (Araneae, Dictynidae). Zoological Journal of the Linnean Society 119: 389-445.

Brignoli, P.M., 1983.A catalogue of the Araneae described between 1940 and 1981. P. Merret, ed. Manchester: Manchester University Press, 755 pp.

Colgan, D.J., \& M.R. Gray, 1992. The genetic structure of the Badumna candida group of species (Araneae, Amaurobioidea). Bulletin of the British arachnological Society 9(3): 86-94.

Davies, V.T., 1998. A revision of the Australian metaltellines (Araneae: Amaurobioidea: Amphinectidae: Metaltellinae). Invertebrate Taxonomy 12: 211-243.

Davies, V.T., \& C. Lambkin, 2000. Wabua, a new spider genus (Araneae: Amaurobioidea: Desidae) from north Queensland, Australia. Memoirs of the Queensland Museum 46(1): 129147.

Dondale, C.D., 1966. The spider fauna (Araneida) of deciduous orchards in the Australian Capital Territory. Australian Journal of Zoology 14: 1157-92.

Downes, M.F., 1993. The life history of Badumna candida (Araneae, Amaurobioidea). Australian Journal of Zoology 41(5): 441-446.

Downes, M.F., 1994a. Tolerance, interattraction and cooperation in the behaviour of the social spider, Phryganoporus candidus (Araneae, Desidae). Bulletin of the British arachnological Society 9(9): 309-317.

Downes, M.F., 1994b. Nest of the social spider Phryganoporus candidus (Araneae: Desidae): Structure, annual growth cycle and host plant relationships. Australian Journal of Zoology 42(2): 237-260.

Forster, R.R., 1970. The spiders of New Zealand. Part III. Otago Museum Bulletin 3: 1-184.

Forster, R.R., \& C.L. Wilton, 1973. The spiders of New Zealand. Part IV. Otago Museum Bulletin 4: 1-309.

Gray, M.R., 1983. The taxonomy of the semi-communal spiders commonly referred to the species Ixeuticus candidus (L. Koch) with notes on the genera Phryganoporus, Ixeuticus and Badumna (Araneae, Amaurobioidea). Proceedings of the Linnean Society of NSW 106: 247-261.
Griswold, C.E., 1990. A revision and phylogenetic analysis of the spider subfamily Phyxelidinae (Araneae, Amaurobiidae). Bulletin of the American Museum of natural History 196: 1206.

Griswold, C.E., J.A. Coddington, N.I. Platnick \& R.R. Forster, 1999. Towards a phylogeny of entelegyne spiders (Araneae, Araneomorphae, Entelegynae). Journal of Arachnology 27: 53 63.

Hickman, V.V., 1967. Some Common Spiders of Tasmania. Hobart: Tasmanian Museum and Art Gallery, pp. 112.

Koch, L., 1872. Die Arachniden Australiens. Vol. I. Nürnberg: Bauer \& Raspe, (3 vols).

Leech, R.,1972. A revision of the Nearctic Amaurobiidae (Arachnida: Araneida). Memoirs of the Entomological Society of Canada 84: 1-182.

Lehtinen, P.T., 1967. Classification of the cribellate spiders and some allied families, with notes on the evolution of the suborder Araneomorpha. Annales Zoologici Fennici 4: 199-468.

Main, B.Y., 1971. The common "colonial" spider Ixeuticus candidus (Koch) and its synonyms (Dictynidae: Araneae). Journal of the Royal Society of Western Australia 54: 119120.

Main, B.Y., 1976. Spiders. Australian Naturalist Library, Collins.

Main, B.Y., \& M.S. Harvey, 1992. Type material of spiders (Chelicerata: Araneae) collected on the Michaelsen and Hartmeyer Expedition of 1905 and lodged in the Western Australian Museum. Records of the Western Australian Museum 15(4): 703-712.

Platnick, N.I., 1989. Advances in Spider Taxonomy 1981-1987: A Supplement to Brignoli's A catalogue of the Araneae Described Between 1940 and 1981 (ed. P. Merrett). Manchester: Manchester University Press.

Ramirez, M.J., 2000. Respiratory system morphology and the phylogeny of haplogyne spiders (Araneae, Araneomorphae). Journal of Arachnology 28: 149-157.

Roewer, C.F., 1954. Katalog der Araneae. Bremen: B. Schünemann, vol. 2 (2 vols).

Simon, E., 1906. Étude sur les Araignées de la section Cribellates. Annales de Societe Entomologique Belgique 50: 284-308.

Simon, E., 1908. Araneae. 1re partie. In Die Fauna SüdwestAustraliens, ed. W. Michaelsen \& R. Hartmeyer, 1(12): 359446. Jena: Fischer Verlag.

Manuscript received 18 July 2000, revised 10 August 2001 and accepted 14 August 2001.

Associate Editor: D.J. Bickel. 